\title{
Analytic approximate solutions to the chemically reactive solute transfer problem with partial slip in the flow of a viscous fluid over an exponentially stretching sheet with suction/blowing
}

https://doi.org/10.1515/eng-2018-0027

Received August 2, 2017; accepted June 24, 2018

\begin{abstract}
This paper analytically investigates the flow as well as the chemically reactive solute transfer problem in a viscous fluid. The motion equations are reduced to a nonlinear ordinary differential equations system using the similarity transformations. The obtained nonlinear differential system is for the first time approximately solved by means of the Optimal Homotopy Asymptotic Method (OHAM). The effects of the partial slip and suction/blowing parameters are analytically analyzed. Some examples are given; the obtained results provides us with a good agreement with the numerical results and reveal that our procedure is effective, accurate and easy to use.
\end{abstract}

Keywords: chemically reactive solute, velocity slip, boundary layer flow, exponential stretching sheet, optimal homotopy asymptotic method

PACS: 47.70.Fw, 47.50.-d, 02.60.-x, 47.11.-j

\section{Introduction}

In all hydraulic machines, the liquid fluids have chemical reactions with the materials from control structures and adjuster devices. Beyond the viscous flow, with all its implications on the energy transfer, the chemical reaction of the liquid fluid affects this transfer in a negative sense. Therefore, the establishment of some relations for the viscous flow, taking into account the chemical reaction, al-

\footnotetext{
*Corresponding Author: Remus-Daniel Ene, Romeo Iosif Negrea: University Politehnica Timişoara, Department of Mathematics, 300006 Timişoara, Romania, E-mail: remus.ene@upt.ro; romeo.negrea@upt.ro

Ilare Bordeaşu: University Politehnica Timişoara, Department of Hydraulic Machines, 300222 Timişoara, Romania, E-mail: ilare.bordeasu@upt.ro
}

lows for the energetic evaluation of the flow, as more appropriate to each situation.

Hydraulic system liquids are used primarily to transmit and distribute forces to various units to be actuated. Liquids are able to do this because they are almost incompressible. Pascal's Law states that pressure applied to any part of a confined liquid is transmitted with undiminished intensity to every other part. Manufacturers of hydraulic devices usually specify the type of liquid best suited for use with their equipment in view of the working conditions, the service required, temperatures expected inside and outside the systems, the pressures the liquid must withstand, the possibilities of corrosion, and other conditions that must be considered. If incompressibility and fluidity were the only qualities required, any liquid that is not too thick could be used in a hydraulic system. One of the most important properties of any hydraulic fluid is its viscosity. Viscosity is internal resistance to flow. A liquid such as gasoline that has a low viscosity flows easily, while a liquid such as tar that has a high viscosity flows slowly. Regardless of its function and design, every hydraulic system has a minimum number of basic components, in addition to a conduit through which the fluid is transmitted. A basic system consists of a pump, reservoir, directional valve, check valve, pressure relieve valve, selector valve, actuator, and filter [1].

In the last few decades, several researchers have studied boundary layer flow, with its practical applications in engineering, electrochemistry and polymer processing technology. Lomen et al. [2] applied a perturbation method to the transport equation for a single reactive chemical with nonlinear (quadratic) rate loss relevant to a soil and water system.

Several researchers applied the shooting method to study the asymptotic behavior of the nonlinear ordinary differential equations that describe the distribution of a solute undergoing a chemical reaction, such as: Zhao et al. 
Table 1: Nomenclature.

\begin{tabular}{|c|c|}
\hline Symbols & Names \\
\hline$u, v$ & Velocity components $(\mathrm{m} / \mathrm{s})$ \\
\hline$x, y$ & Cartesian coordinates $(m)$ \\
\hline$v$ & Fluid kinematic viscosity $\left(\mathrm{m}^{2} / \mathrm{s}\right)$ \\
\hline C & Concentration of the chemically reactive species \\
\hline$C_{\infty}$ & Concentration far away from the sheet \\
\hline \multirow[t]{2}{*}{$D$} & Brownian diffusion coefficient $\left(\mathrm{m}^{2} / \mathrm{s}\right)$ \\
\hline & (Molecular diffusivity of the chemically reactive species) \\
\hline \multirow[t]{2}{*}{$k$} & Chemical reaction parameter \\
\hline & (Variable rate of chemical conversion of the first-order irreversible reaction) \\
\hline$U$ & Stretching velocity $(\mathrm{m} / \mathrm{s})$ \\
\hline$C_{w}$ & Concentration on the sheet \\
\hline$U_{0}, C_{0}$ & Reference velocity and reference concentration \\
\hline$N$ & Velocity slip factor \\
\hline$S c$ & Schmidt number \\
\hline$\beta$ & Reaction rate parameter \\
\hline$\lambda$ & Velocity slip parameter \\
\hline$N_{G}$ & Dimensionless entropy number \\
\hline$R e$ & Reynolds number \\
\hline$B_{r}$ & Brinkman number \\
\hline$T_{\infty}$ & Environmental temperature $(K)$ \\
\hline$\chi, \lambda_{1}$ & Dimensionless constant parameters \\
\hline$\Omega$ & Dimensionless temperature difference \\
\hline
\end{tabular}

[3], Bhattacharyya and Layek [4], Bhattacharyya [5], and Mukhopadhyay [6-10].

An approximate solution to a differential equation in the form of an analytic expression can be found by the method of series, the method of small parameters, the method of successive approximations, the perturbation method, the Ritz and Galerkin method, among others. Each of these methods defines one or more infinite processes that under certain conditions can be used to obtain an exact solution to a problem. Termination of the process after a finite number of steps yields an approximate solution. In fact, an analytical method gives a solution in the form of symbols, i.e. closed form solution. A numerical method gives solution at certain points only. Hence, the analytical solution denotes an exact solution that can be used to study the behavior of the system with varying properties.

In the last years, two interesting methods to obtain an approximate analytical solution were used for solving more sophisticated PDEs. The Homotopy Analysis Method (HAM) was developed by Liao [11] who utilized the idea of homotopy in topology. The Optimal Homotopy Asymptotic Method (OHAM) was introduced by Marinca and Herisanu
[12]. An advantage of OHAM is that it does not need to identify the $\hbar$ - curve, and the control and adjustment of the convergence region is provided in a conventional way. Furthermore, the OHAM has a built in convergence criteria similar to HAM but with a greater degree of flexibility.

Along with the analytical methods, some computational methods to solve nonlinear problems from engineering and computer sciences have been developed by Li et al. [17-24], Guo et al. [25], Korda et al. [26].

In this paper, the optimal homotopy asymptotic method (OHAM) [12-16] is applied to obtain accurate, effective analytic approximate solutions. The quality of the approximate solutions is investigated using two important statistical tests: the Bartlett test and the Durbin-Wattson test. Our procedure does not depend upon small or large parameters, and provides us with a simple way to optimally control the convergence of the approximate solutions. 


\section{Equations of motion}

We analyze the two-dimensional flow of an incompressible viscous fluid past a flat sheet in the half-plane $y>0$. As in [9], two equal and opposite forces are applied along the $x$ - and $y$ - axis so that the wall is stretched keeping the origin fixed. The schematic diagram of the physical model is presented in Fig. 1. Also, the continuity, momentum and

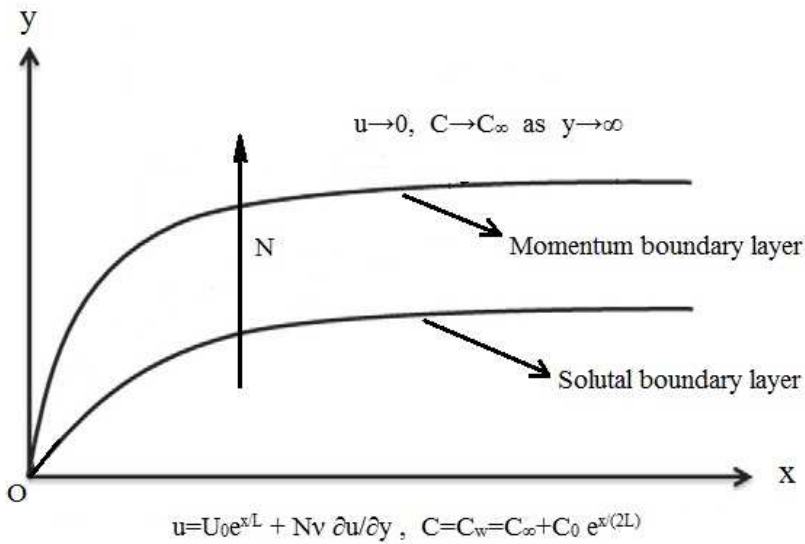

Figure 1: Schematic diagram of the physical model.

concentration equations governing such a type of the flow are given as [9]:

$$
\begin{gathered}
\frac{\partial u}{\partial x}+\frac{\partial v}{\partial y}=0 \\
u \frac{\partial u}{\partial x}+v \frac{\partial u}{\partial y}=v \frac{\partial^{2} u}{\partial y^{2}}, \\
u \frac{\partial C}{\partial x}+v \frac{\partial C}{\partial y}=D \frac{\partial^{2} C}{\partial y^{2}}-k\left(C-C_{\infty}\right),
\end{gathered}
$$

where $k=\frac{1}{2} k_{0} e^{x / L}$.

The physical initial/boundary conditions for this case are similar as in [9], [27, 28]:

$$
\begin{aligned}
& y=0: u=U+N v \frac{\partial u}{\partial y}, \quad v=-V(x), C=C_{w}, \\
& y \rightarrow \infty: u \rightarrow 0, \quad C \rightarrow C_{\infty},
\end{aligned}
$$

where $U=U_{0} e^{x / L}, C_{w}=C_{\infty}+C_{0} e^{x /(2 L)}$, $N=N_{1} e^{-x /(2 L)}$ is the velocity slip factor, which changes with $x$ (at $N=0$, no-slip case is observed), $N_{1}$ is the initial value of the velocity slip factor, $V(x)>0$ is the velocity of suction, $V(x)<0$ is the velocity of blowing, $V(x)=V_{0} e^{x /(2 L)}$ is the velocity on the wall, $V_{0}$ is the initial strength of suction, and $k_{0}$ is a constant having the same dimension as $k$.
Using the similarity transformations:

$$
\begin{aligned}
& \eta=\sqrt{\frac{U_{0}}{2 v L}} e^{x /(2 L)} y, \quad u=U_{0} e^{x /(2 L)} f^{\prime}(\eta), \\
& v=-\sqrt{\frac{v U_{0}}{2 L}} e^{x /(2 L)}\left[f(\eta)+\eta f^{\prime}(\eta)\right], \\
& C=C_{\infty}+C_{0} e^{x /(2 L)} \varphi(\eta)
\end{aligned}
$$

and substituting Eq. (4) into Eqs. (1) and (2), the governing equations become:

$$
\begin{gathered}
f^{\prime \prime \prime}+f f^{\prime \prime}-2\left(f^{\prime}\right)^{2}=0, \\
\varphi^{\prime \prime}+S c\left(f \varphi^{\prime}-f^{\prime} \varphi-\beta \varphi\right)=0,
\end{gathered}
$$

where $S c=v / D$ and $\beta=k_{0} L / U_{0}$.

The initial/boundary conditions Eq. (3) become:

$$
\begin{aligned}
& f(0)=S, \quad f^{\prime}(0)=1+\lambda f^{\prime \prime}(0), \quad \varphi(0)=1, \\
& f^{\prime} \rightarrow 0, \quad \varphi \rightarrow 0 \text { for } \eta \rightarrow \infty,
\end{aligned}
$$

where $S=V_{0} \sqrt{\frac{2 L}{U_{0} v}}>0(<0)$ is the suction (or blowing) parameter, $\lambda=N_{1} \sqrt{\frac{U_{0} v}{2 L}}$ and the prime denotes differentiation with respect to $\eta$.

Remark: In Mukhopadhyay et al. [9] there is a major error in the Equation of concentration. By a simple computation the correct equation of concentration is Eq. (6), rather than

$$
\varphi^{\prime \prime}+S c\left(f \varphi^{\prime}-f^{\prime} \varphi+\beta \varphi\right)=0,
$$

as in Mukhopadhyay et al. [9]. Therefore, in our paper the equation of concentration Eq. (6) is correct.

\section{Basic ideas of the optimal homotopy asymptotic method}

In Marinca and Herisanu [12], the optimal homotopy asymptotic method is employed to compute analytical approximate solutions for equations of the general form:

$$
L(F(y))+N(F(y))=0,
$$

subject to the boundary / initial conditions of the type

$$
B\left(F(y), \frac{d F(y)}{d y}\right)=0 .
$$


For the flow of viscous fluid determined by Eqs. (5) and (6) with initial/boundary conditions (7), the corresponding operators $L, N$ and $B$ will be introduced in the next section.

Using the principle of OHAM [12-16], we are going to construct the following homotopy:

$$
\mathcal{H}\left[L(F(y, p)), H\left(y, C_{i}\right), N(F(y, p))\right],
$$

where $p \in[0,1]$ is the embedding parameter, $L$ is a linear operator, and $H\left(y, C_{i}\right) \neq 0$ is an auxiliary convergencecontrol function. $H$ is a function of the variable $y$ and of the parameters $C_{1}, C_{2}, \ldots, C_{s}$.

The homotopy (10) satisfies the following properties:

$$
\begin{array}{r}
\mathcal{H}\left[L(F(y, 0)), H\left(y, C_{i}\right), N(F(y, 0))\right]= \\
=L(F(y, 0))=L\left(F_{0}(y)\right), \\
\mathcal{H}\left[L(F(y, 1)), H\left(y, C_{i}\right), N(F(y, 1))\right]= \\
=H\left(y, C_{i}\right) N(F(y, 1)) .
\end{array}
$$

If the function $F$ has the expression:

$$
F(y, p)=F_{0}(y)+p F_{1}\left(y, C_{i}\right)
$$

by substituting this expression in the homotopy Eq. (10) we obtain the following relation:

$$
\mathcal{H}\left[L(F(y, p)), H\left(y, C_{i}\right), N(F(y, p))\right]=0 .
$$

If we choose the following particular expression of the operator $\mathcal{H}$ :

$$
\begin{array}{r}
\mathcal{H}\left[L(F(y, p)), H\left(y, C_{i}\right), N(F(y, p))\right]= \\
=L\left(F_{0}(y)\right)+p\left[L\left(F_{1}\left(y, C_{i}\right)\right)-H\left(y, C_{i}\right) N\left(F_{0}(y)\right)\right],
\end{array}
$$

we can obtain the governing equations of $F_{0}(y)$ and $F_{1}\left(y, C_{i}\right)$ by equating the coefficients of $p^{0}$ and $p^{1}$, respectively:

$$
\begin{gathered}
L\left(F_{0}(y)\right)=0, \quad B\left(F_{0}(y), \frac{d F_{0}(y)}{d y}\right)=0, \\
L\left(F_{1}\left(y, C_{i}\right)\right)=H\left(y, C_{i}\right) N\left(F_{0}(y)\right), \\
B\left(F_{1}\left(y, C_{i}\right), \frac{d F_{1}\left(y, C_{i}\right)}{d y}\right)=0, \quad i=1,2, \ldots, s .
\end{gathered}
$$

$F_{0}(y)$ can be readily found by solving the linear Eq. (16). In order to compute $F_{1}\left(y, C_{i}\right)$ by Eq. (17), we take into account the fact that the nonlinear operator $N$ has the general form:

$$
N\left(F_{0}(y)\right)=\sum_{i=1}^{n} h_{i}(y) g_{i}(y),
$$

where $n$ is a positive integer, and $h_{i}(y)$ and $g_{i}(y)$ are known functions that depend on $F_{0}(y)$ and on $N$.

The general solution of the nonhomogeneous linear equation (17) is obtained by summing the general solution of the corresponding homogeneous equation and a particular solution of the nonhomogeneous equation. Unfortunately, the computation of such a particular solution is not possible in most cases, so the computation of the function $F_{1}\left(y, C_{i}\right)$, introduced as a third modified version of Optimal Homotopy Asymptotic Method in [12], consists of the following steps:

- We consider the $F_{1}\left(y, C_{i}\right)$ of the form:

$$
F_{1}\left(y, C_{i}\right)=\sum_{i=1}^{m} H_{i}\left(y, h_{j}(y), C_{j}\right) g_{i}(y), \quad j=1, \ldots, s,
$$

or

$$
\begin{aligned}
& F_{1}\left(y, C_{i}\right)=\sum_{i=1}^{m} H_{i}\left(y, g_{j}(y), C_{j}\right) h_{i}(y), j=1, \ldots, s, \\
& B\left(F_{1}\left(y, C_{i}\right), \frac{d F_{1}\left(y, C_{i}\right)}{d y}\right)=0 .
\end{aligned}
$$

The above expressions of $H_{i}\left(y, h_{j}(y), C_{j}\right)$ contain linear combinations of the functions $h_{j}, j=1, \ldots, s$. They also contain the parameters $C_{j}, j=1, \ldots, s$. The summation limit $m$ is an arbitrary positive integer number.

- Taking into account Eq. (13), the first-order analytical approximate solution of Eqs. (8) - (9) is:

$$
\bar{F}\left(y, C_{i}\right)=F(y, 1)=F_{0}(y)+F_{1}\left(y, C_{i}\right) .
$$

- Finally, the convergence-control parameters $C_{1}$, $C_{2}, \ldots, C_{s}$ can be optimally computed by means of various methods, such as: the least square method, the Galerkin method, the collocation method, the Kantorowich method, or the weighted residual method.

With these parameters known, the first-order approximate solution (21) is well-determined.

\section{Application of the OHAM to the chemically reactive solute transfer problem}

Now we are going to apply our procedure to obtain approximate solutions of Eqs. (5) and (6) with the initial / boundary conditions Eq. (7). 
For this purpose, in the case of the nonlinear equation Eq. (5), we choose the linear operator of the form:

$$
L_{f}(\eta)=f^{\prime \prime \prime}(\eta)+\frac{3 K}{K \eta+1} f^{\prime \prime}(\eta),
$$

where $K$ is an unknown positive parameter and will be determined later.

As in Marinca and Herisanu [12], it is easy to show that the linear operator is not unique.

The initial approximation $f_{0}(\eta)$ can be obtained from the following problem:

$$
\begin{aligned}
& f_{0}^{\prime \prime \prime}(\eta)+\frac{3 K}{K \eta+1} f_{0}^{\prime \prime}(\eta)=0, \\
& f_{0}(0)=S, \quad f_{0}^{\prime}(0)=1+\lambda f_{0}^{\prime \prime}(0), \quad f_{0}^{\prime}(\infty)=0,
\end{aligned}
$$

which has the solution

$$
f_{0}(\eta)=S+\frac{1}{K(2 \lambda K+1)}-\frac{1}{K(2 \lambda K+1)} \cdot \frac{1}{K \eta+1} .
$$

The nonlinear operator $N_{f}(\eta)$, corresponding to nonlinear differential Eq. (5), is defined by:

$$
N_{f}(\eta)=-\frac{3 K}{K \eta+1} f^{\prime \prime}+f f^{\prime \prime}-2\left(f^{\prime}\right)^{2} .
$$

For the initial approximation $f_{0}(\eta)$ given by Eq. (24), the nonlinear operator Eq. (25) becomes:

$$
\begin{aligned}
& N_{f_{0}}(\eta)=-\frac{3 K}{K \eta+1} f_{0}^{\prime \prime}+f_{0} f_{0}^{\prime \prime}-2\left(f_{0}^{\prime}\right)^{2}= \\
& =-\frac{2(K S+1)}{(2 K \lambda+1)^{2}} \cdot \frac{1}{(K \eta+1)^{3}}+\frac{6 K^{2}}{2 K \lambda+1} \cdot \frac{1}{(K \eta+1)^{4}} .
\end{aligned}
$$

Comparing Eqs. (26) and (18), one can write:

$$
\begin{aligned}
& h_{1}(\eta)=-\frac{2(K S+1)}{(2 K \lambda+1)^{2}}, \quad g_{1}(\eta)=\frac{1}{(K \eta+1)^{3}}, \\
& h_{2}(\eta)=\frac{6 K^{2}}{2 K \lambda+1}, \quad g_{2}(\eta)=\frac{1}{(K \eta+1)^{4}} .
\end{aligned}
$$

The function $f_{1}(\eta)$ given by Eq. (19) becomes:

$$
f_{1}\left(\eta, C_{i}\right)=H_{1}\left(\eta, C_{i}\right) \frac{1}{(K \eta+1)^{3}}+H_{2}\left(\eta, C_{i}\right) \frac{1}{(K \eta+1)^{4}},
$$

where we have freedom to choose a lot of possibilities for the unknown functions $H_{i}, i=1,2$, as follows (see Marinca and Herisanu [12]):

$$
\begin{aligned}
& H_{1}\left(\eta, C_{i}\right)=A_{1}(K \eta+1)^{3}-\frac{A_{2}}{K(2 K \lambda+1)}(K \eta+1)^{2}+ \\
& +C_{1}(K \eta+1)+C_{2}, \\
& H_{2}\left(\eta, C_{i}\right)=C_{3}+\sum_{i=1}^{7} \frac{C_{i+3}}{(K \eta+1)^{i}}+\frac{C_{11} \eta^{2}}{(K \eta+1)^{10}} .
\end{aligned}
$$

Substituting Eq. (29) into Eq. (28) we have:

$$
\begin{aligned}
& f_{1}\left(\eta, C_{i}\right)=A_{1}-\frac{1}{K(2 K \lambda+1)} \frac{A_{2}}{K \eta+1}+ \\
& +\sum_{i=1}^{10} \frac{C_{i}}{(K \eta+1)^{i+1}}+\frac{C_{11} \eta^{2}}{(K \eta+1)^{14}},
\end{aligned}
$$

where

$$
\begin{gathered}
A_{1}=\frac{A_{2}}{K(2 K \lambda+1)}-\sum_{i=1}^{10} C_{i}, \\
A_{2}=K \cdot \sum_{i=1}^{10}(i+1) C_{i}+\lambda K^{2} \cdot \sum_{i=1}^{10}(i+1)(i+2) C_{i}+2 \lambda C_{11} .
\end{gathered}
$$

The first-order approximate solution given by Eq. (21) is obtained from Eqs. (24) and (30):

$$
\begin{aligned}
& \bar{f}\left(\eta, C_{i}\right)=f_{0}(\eta)+f_{1}\left(\eta, C_{i}\right)=S+A_{1}+\frac{1}{K(2 K \lambda+1)}- \\
& -\frac{1}{K(2 K \lambda+1)} \frac{1+A_{2}}{K \eta+1}+\sum_{i=1}^{10} \frac{C_{i}}{(K \eta+1)^{i+1}}+\frac{C_{11} \eta^{2}}{(K \eta+1)^{14}} .
\end{aligned}
$$

In this way, we can find other solutions as well.

For Eq. (6) with initial/boundary condition given by Eq. (7) (for the unknown function $\varphi$ ), the expression for the linear operator $L_{\varphi}(\eta)$ is chosen as:

$$
L_{\varphi}(\eta)=\varphi^{\prime \prime}+\frac{2}{K_{1} \eta+1} \varphi^{\prime},
$$

where $K_{1}>0$ is an unknown parameter at this moment.

Eq. (16) can be written in the form:

$$
\varphi_{0}^{\prime \prime}+\frac{2}{K_{1} \eta+1} \varphi_{0}^{\prime}=0, \quad \varphi_{0}(0)=1, \quad \varphi_{0}(\infty)=0
$$

and has the solution

$$
\varphi_{0}(\eta)=\frac{1}{K_{1} \eta+1} .
$$

The nonlinear operator $N_{\varphi}$ corresponding to the unknown function $\varphi$ is obtained from the expression Eq. (6) in the form:

$$
N_{\varphi}(\eta)=-\frac{2}{K_{1} \eta+1} \varphi^{\prime}+S c\left(f \varphi^{\prime}-f^{\prime} \varphi-\beta \varphi\right) .
$$

For the initial approximation $\varphi_{0}(\eta)$ given by Eq. (34), the nonlinear operator Eq. (35) becomes:

$$
\begin{aligned}
& N_{\varphi_{0}}(\eta)=-\frac{S c \beta}{K_{1} \eta+1}-K_{1} S c\left[S+\frac{1}{K(2 K \lambda+1)}\right] \times \\
& \times \frac{1}{\left(K_{1} \eta+1\right)^{2}}+\frac{2 K_{1}}{\left(K_{1} \eta+1\right)^{3}}-\frac{S c}{2 \lambda K+1} \frac{1}{(K \eta+1)^{2}} \frac{1}{K_{1} \eta+1}+ \\
& +\frac{S c K_{1}}{K(2 \lambda K+1)} \frac{1}{K \eta+1} \frac{1}{\left(K_{1} \eta+1\right)^{2}} .
\end{aligned}
$$

By comparing the Eqs. (18) and (36) one can get:

$$
h_{1}^{\star}(\eta)=-S c \beta, g_{1}^{\star}(\eta)=\frac{1}{K_{1} \eta+1},
$$

$$
\begin{gathered}
h_{2}^{\star}(\eta)=-K_{1} S c\left[S+\frac{1}{K(2 K \lambda+1)}\right], g_{2}^{\star}(\eta)=\frac{1}{\left(K_{1} \eta+1\right)^{2}}, \\
h_{3}^{\star}(\eta)=2 K_{1}, \quad g_{3}^{\star}(\eta)=\frac{1}{\left(K_{1} \eta+1\right)^{3}},
\end{gathered}
$$




$$
\begin{gathered}
h_{4}^{\star}(\eta)=\frac{S c K_{1}}{K(2 \lambda K+1)}, \quad g_{4}^{\star}(\eta)=\frac{1}{K \eta+1} \frac{1}{\left(K_{1} \eta+1\right)^{2}}, \\
h_{5}^{\star}(\eta)=-\frac{S c}{2 \lambda K+1}, \quad g_{5}^{\star}(\eta)=\frac{1}{(K \eta+1)^{2}} \frac{1}{K_{1} \eta+1} .
\end{gathered}
$$

The first approximation $\varphi_{1}\left(\eta, D_{i}\right)$, given by Eq. (19), becomes:

$$
\begin{aligned}
& \varphi_{1}\left(\eta, D_{i}\right)=H_{1}^{\star}\left(\eta, D_{i}\right) \frac{1}{K_{1} \eta+1}+H_{2}^{\star}\left(\eta, D_{i}\right) \frac{1}{\left(K_{1} \eta+1\right)^{2}}+ \\
& +H_{3}^{\star}\left(\eta, D_{i}\right) \frac{1}{\left(K_{1} \eta+1\right)^{3}}+H_{4}^{\star}\left(\eta, D_{i}\right) \frac{1}{K \eta+1} \frac{1}{\left(K_{1} \eta+1\right)^{2}}+ \\
& +H_{5}^{\star}\left(\eta, D_{i}\right) \frac{1}{(K \eta+1)^{2}} \frac{1}{K_{1} \eta+1},
\end{aligned}
$$

where $D_{i}$ are unknown parameters, and the unknown auxiliary functions $H_{1}^{\star}\left(\eta, D_{i}\right), \ldots, H_{5}^{\star}\left(\eta, D_{i}\right)$ can be chosen in the form:

$$
\begin{aligned}
& H_{1}^{\star}\left(\eta, D_{i}\right)=M_{1}, H_{2}^{\star}\left(\eta, D_{i}\right)=D_{1}, H_{3}^{\star}\left(\eta, D_{i}\right)=D_{2}, \\
& H_{4}^{\star}\left(\eta, D_{i}\right)=D_{4}\left(K_{1} \eta+1\right)^{2}+\frac{D_{5}}{\left(K_{1} \eta+1\right)^{3}}+\frac{D_{6}}{\left(K_{1} \eta+1\right)^{4}}+ \\
& +\frac{D_{7}}{\left(K_{1} \eta+1\right)^{5}}+\frac{D_{8} \eta^{2}}{\left(K_{1} \eta+1\right)^{6}}, \\
& H_{5}^{\star}\left(\eta, D_{i}\right)=D_{9}\left(K_{1} \eta+1\right)+\frac{D_{10}}{\left(K_{1} \eta+1\right)^{8}}+ \\
& +\frac{D_{11}}{\left(K_{1} \eta+1\right)^{9}}+\frac{D_{12} \eta^{2}}{\left(K_{1} \eta+1\right)^{10}}
\end{aligned}
$$

where $M_{1}=-\sum_{i=1}^{7} D_{i}-\sum_{i=9}^{11} D_{i}$.

Substituting Eq. (39) into Eq. (38) one can get:

$$
\begin{aligned}
& \varphi_{1}\left(\eta, D_{i}\right)=\frac{M_{1}}{K_{1} \eta+1}+\sum_{i=1}^{3} \frac{D_{i}}{\left(K_{1} \eta+1\right)^{i+1}}+ \\
& +\left[D_{4}+\sum_{i=5}^{7} \frac{D_{i}}{\left(K_{1} \eta+1\right)^{i}}+\frac{D_{8} \eta^{2}}{\left(K_{1} \eta+1\right)^{8}}\right] \cdot \frac{1}{K \eta+1}+ \\
& +\left[D_{9}+\sum_{i=10}^{11} \frac{D_{i}}{\left(K_{1} \eta+1\right)^{i-1}}+\frac{D_{12} \eta^{2}}{\left(K_{1} \eta+1\right)^{11}}\right] \cdot \frac{1}{(K \eta+1)^{2}} .
\end{aligned}
$$

The first-order approximate solution given by Eq. (21) is obtained from Eqs. (34) and (40):

$$
\begin{aligned}
& \bar{\varphi}\left(\eta, D_{i}\right)=\varphi_{0}(\eta)+\varphi_{1}\left(\eta, D_{i}\right)= \\
& =\frac{M_{1}+1}{K_{1} \eta+1}+\sum_{i=1}^{3} \frac{D_{i}}{\left(K_{1} \eta+1\right)^{i+1}}+ \\
& +\left[D_{4}+\sum_{i=5}^{7} \frac{D_{i}}{\left(K_{1} \eta+1\right)^{i}}+\frac{D_{8} \eta^{2}}{\left(K_{1} \eta+1\right)^{8}}\right] \cdot \frac{1}{K \eta+1}+ \\
& +\left[D_{9}+\sum_{i=10}^{11} \frac{D_{i}}{\left(K_{1} \eta+1\right)^{i-1}}+\frac{D_{12} \eta^{2}}{\left(K_{1} \eta+1\right)^{11}}\right] \cdot \frac{1}{(K \eta+1)^{2}} .
\end{aligned}
$$

\section{Results and discussions}

In order to prove the accuracy of the obtained results, we will determine the convergence-control parameters $K$, $C_{i}, K_{1}$ and $D_{i}$, which appear in Eqs. (31) and (41), by the least square means method. In this way, the convergencecontrol parameters are optimally determined, and the firstorder approximate solutions become known for different values of the known parameters $S, \lambda, \beta$ and $S c$. In what follows, we illustrate the accuracy of the OHAM, comparing previously obtained approximate solutions with the numerical integration results, computed by means of the shooting method combined with fourth-order RungeKutta method using Wolfram Mathematica 6.0 software. For some values of the parameters $S, \lambda, \beta$ and $S c$ we are going to determine the approximate solutions.

Example 5.1 For the first case, we consider $S=-0.5$, $\lambda=0.1$. For Eq. (31), following the procedure described above, the first-order approximate solution is obtained:

$$
\begin{aligned}
& \bar{f}(\eta)=0.2032088596-\frac{1519.5683116346}{(1+0.0823625270 \eta)^{11}}+ \\
& +\frac{7190.3303368698}{(1+0.0823625270 \eta)^{10}}-\frac{14201.5043202427}{(1+0.0823625270 \eta)^{9}}+
\end{aligned}
$$

$$
\begin{gathered}
+\frac{14745.8618922473}{(1+0.0823625270 \eta)^{8}}-\frac{7974.2698036553}{(1+0.0823625270 \eta)^{7}}+ \\
+\frac{1366.3687483408}{(1+0.0823625270 \eta)^{6}}+\frac{800.5049144492}{(1+0.0823625270 \eta)^{5}}- \\
-\frac{521.3976095484}{(1+0.0823625270 \eta)^{4}}+\frac{127.0468163267}{(1+0.0823625270 \eta)^{3}}- \\
-\frac{14.7524994058}{(1+0.0823625270 \eta)^{2}}+\frac{0.6766273933}{1+0.0823625270 \eta}{ }^{+} \\
+\frac{0.3089556785 \eta^{2}}{(1+0.0823625270 \eta)^{14}} .
\end{gathered}
$$


Now, in this case we give the four approximate solutions $\bar{\varphi}(\eta)$ for concentration obtained from Eq. (41), for different value of the Schmidt parameter $S c$ :

$\mathbf{a}_{1}$ ) the reaction rate parameter $\beta=0.1$, the Schmidt parameter $S c=0.7$.

$$
\begin{aligned}
& \bar{\varphi}(\eta)=-\frac{3.9816029319}{(1+0.1646305546 \eta)^{4}}+ \\
& +\frac{4.0335307886}{(1+0.1646305546 \eta)^{3}}-\frac{0.1534745401}{(1+0.1646305546 \eta)^{2}}- \\
& -\frac{5.0593327058}{1+0.1646305546 \eta}+[1.3512198945- \\
& -\frac{3.1027133726}{(1+0.1646305546 \eta)^{10}}+\frac{1.3429468640}{(1+0.1646305546 \eta)^{9}}+ \\
& \left.+\frac{0.0671762598 \eta^{2}}{(1+0.1646305546 \eta)^{11}}\right] \cdot \frac{1}{(1+0.0823625270 \eta)^{2}}+ \\
& +\left[2.5267225444+\frac{2.9456463231}{(1+0.1646305546 \eta)^{7}}+\right. \\
& +\frac{2.9607435234}{(1+0.1646305546 \eta)^{6}}-\frac{1.8636863877}{(1+0.1646305546 \eta)^{5}}+ \\
& \left.+\frac{0.6559223088 \eta^{2}}{(1+0.1646305546 \eta)^{8}}\right] \cdot \frac{1}{1+0.0823625270 \eta} .
\end{aligned}
$$

More results are given in Appendix 6.

\section{Remarks:}

1. The errors between the numerical solution and the approximate solution are not natural errors, but errors derived from observations. If the errors come just from the computer approximation of the real numbers, then we can call them real errors; otherwise, they are residual errors, and some statistical tests are necessary to check if these errors hedge or hide some others terms (functions). Then, the obtained approximate solution is not the best solution. For this reason, two statistical tests are usually used: the test of homoscedasticity and the test of autocorrelation. We compute the Durbin-Wattson test for autocorrelation, and the Bartlett test for homoscedasticity:

$$
\begin{gathered}
d=\sum_{i=1}^{2 N}\left(\varepsilon_{i}-\varepsilon_{i-1}\right)^{2} / \sum_{i=1}^{N} \varepsilon_{i}^{2} \text { (Durbin-Wattson test), } \\
F_{\text {calc }}=\sum_{i=1}^{N / 2} \varepsilon_{i}^{2} / \sum_{i=[N / 2]+1}^{N} \varepsilon_{i}^{2} \text { (Bartlett test). }
\end{gathered}
$$

For approximate solutions (Eq. (31)), in the case $S=$ 0 the errors pass both tests, in the other cases $(S=1$ or
$S=-1$ ) some small positive autocorrelations are obtained. This behavior can be explained by the greater values of the $\beta$ coefficient of rate reaction, which is difficult to be cached by a numerical solution but is rigorously included in an approximate solution.

2. The approximate solution should have the simplest form; i.e. should have an optimal number of parameters. A good criteria to find the optimal numbers of parameters is that given by Akaike [29] (in 1974). Given a collection of models for the dates, Akaike Informational Criterion (AIC) estimates the quality of each model, relative to each of the other models. For the least squares estimation, the AIC value of the model is the following:

$$
A I C(k)=n \log \left(\frac{R S S}{n}\right)+2 k,
$$

where RSS = residual sum of errors, $k$ - the number of parameters, $n$ - the number of observations. The best model is that which finds the minimum value of $A I C(k)$.

In our case, we compute the value of AIC for $k=$ $12,14,16$ parameters and find the minimum value for $k=12$.

Tables 2 - 4 show the comparisons between the OHAM approximate solutions $\left(\bar{f}_{O H A M}, \bar{f}_{O H A M}^{\prime}\right)$ given by Eqs. (42), (49), (50), and numerical integration, for $\lambda=0.1$ and different values of the suction/blowing coefficient $S$. Table 5 provides a comparison between the OHAM approximate solutions $\bar{\varphi}_{O H A M}$ (concentration) given by Eqs. (43), (46), (47) and (48) for the flow with blowing, i.e. $S=-0.5$, and numerical results for $\lambda=0.1, \beta=0.1$ and different values of the Schmidt coefficient $S c$.

On the other hand, in Tables 6 and 7 the comparisons between the skin-friction coefficient $f^{\prime \prime}(0)$ and the limit value $f(\infty)$, respectively, are presented, obtained from Eqs. (42), (49) and (50) and numerical results, for $\lambda=0.1$ and different values of the suction/blowing coefficient $S$.

A comparison between the mass transfer coefficient $\varphi^{\prime}(0)$ obtained from Eqs. (43), (46), (47) and (48) with numerical results, for the flow with blowing $(S=-0.5)$, and for the flow with suction $(S=0.5)$, respectively, are presented in Tables 8 and 9, respectively.

If the analytical approximate solutions $\bar{f}$ and $\bar{\varphi}$ are given by the Eqs (31) and (41) respectively, then the residuals from Eqs. (5) and (6) respectively are:

$$
R_{\bar{f}}(\eta)=\bar{f}^{\prime \prime \prime}(\eta)+\bar{f}(\eta) \bar{f}^{\prime \prime}(\eta)-2\left(\bar{f}^{\prime}(\eta)\right)^{2}
$$

and

$$
R_{\bar{\varphi}}(\eta)=\bar{\varphi}^{\prime \prime}(\eta)+S c \cdot\left(\bar{f}(\eta) \bar{\varphi}^{\prime}(\eta)-\bar{f}^{\prime}(\eta) \bar{\varphi}(\eta)-\beta \bar{\varphi}(\eta)\right) .
$$


Table 2: Comparison between OHAM results $\left(\bar{f}, \bar{f}^{\prime}\right)$ given by Eq. (42) and numerical results, for $S=-0.5, \lambda=0.1$ (relative errors: $\epsilon_{f}=\left|f_{\text {numerical }}-\bar{f}_{O H A M}\right|, \epsilon_{f^{\prime}}=\left|f_{\text {numerical }}^{\prime}-\bar{f}_{O H A M}^{\prime}\right|$ )

\begin{tabular}{|c|c|c|c|c|c|c|}
\hline$\eta$ & $f_{\text {numerical }}$ & $\begin{array}{l}\bar{f}_{O H A M} \\
\text { given by } \\
\text { Eq. }(42)\end{array}$ & $\epsilon_{f}$ & $f_{\text {numerical }}^{\prime}$ & $\begin{array}{l}\bar{f}_{O H A M}^{\prime} \\
\text { from } \\
\text { Eq. (42) }\end{array}$ & $\epsilon_{f^{\prime}}$ \\
\hline 0 & -0.5 & -0.5 & 0 & 0.9077773126 & 0.9077773227 & $1.01 \cdot 10^{-8}$ \\
\hline $4 / 5$ & 0.0074909817 & 0.0074077629 & $8.32 \cdot 10^{-5}$ & 0.4327638766 & 0.4327294709 & $3.44 \cdot 10^{-5}$ \\
\hline $8 / 5$ & 0.2611017466 & 0.2611117146 & $9.96 \cdot 10^{-6}$ & 0.2266414650 & 0.2268106116 & $1.69 \cdot 10^{-4}$ \\
\hline $12 / 5$ & 0.3966238195 & 0.3966963649 & $7.25 \cdot 10^{-5}$ & 0.1228892931 & 0.1228628361 & $2.64 \cdot 10^{-5}$ \\
\hline $16 / 5$ & 0.4696414089 & 0.4696460798 & $4.67 \cdot 10^{-6}$ & 0.0648025592 & 0.0646955746 & $1.06 \cdot 10^{-4}$ \\
\hline $20 / 5$ & 0.5063210253 & 0.5062657176 & $5.53 \cdot 10^{-5}$ & 0.0296392786 & 0.0296092095 & $3.006 \cdot 10^{-5}$ \\
\hline $24 / 5$ & 0.5204156378 & 0.5203725140 & $4.31 \cdot 10^{-5}$ & 0.0072024341 & 0.0072543060 & $5.18 \cdot 10^{-5}$ \\
\hline $28 / 5$ & 0.5198883866 & 0.5198975191 & $9.13 \cdot 10^{-6}$ & -0.0074991548 & -0.0074318639 & $6.72 \cdot 10^{-5}$ \\
\hline $32 / 5$ & 0.5097627316 & 0.5098134397 & $5.07 \cdot 10^{-5}$ & -0.0171195125 & -0.0170879834 & $3.15 \cdot 10^{-5}$ \\
\hline $36 / 5$ & 0.4934363158 & 0.4934931771 & $5.68 \cdot 10^{-5}$ & -0.0232005067 & -0.0232154713 & $1.49 \cdot 10^{-5}$ \\
\hline $40 / 5$ & 0.4733209745 & 0.4733525235 & $3.15 \cdot 10^{-5}$ & -0.0267255573 & -0.0267697975 & $4.42 \cdot 10^{-5}$ \\
\hline
\end{tabular}

An error analysis by computing the integral of the square residuals given by Eqs. (44) and (45) is presented in Table 10.

The profiles of the stream function $\bar{f}(\eta)$ given by Eqs. (42), (49), (50) are depicted in Fig. 2 for $\lambda=0.1$. We observe that the stream function $\bar{f}(\eta)$ increases with an increase of the suction/blowing coefficient $S$.

From Fig. 3 the velocity profiles $\bar{f}^{\prime}(\eta)$ decreases with an increasing of the suction/blowing coefficient $S$.

Fig. 4 presents the variation of the shear-stress function $\bar{f}^{\prime \prime}(\eta)$ obtained from Eqs. (42), (49) and (50). It is noticed that the shear-stress function is increasing against an increase of the suction/blowing coefficient.

On the other hand, in Fig. 5 the profiles of the concentration function $\bar{\varphi}(\eta)$ for the flow with blowing $(S=-0.5)$ and suction $(S=0.5)$ are plotted. It is noticed that the concentration function is decreasing with respect to an increase of the Schmidt coefficient Sc. For fixed value of the slip parameter $\lambda$ and the reaction rate parameter $\beta$, from Fig. 5 we can notice that the concentration function $\bar{\varphi}(\eta)$ decreases against an increase of the suction/blowing coefficient $S$, for all the values of the Schmidt coefficient $S c$.

The effect of the slip parameter $\lambda$ on the chemically reactive solute flow is presented in Fig. 6 for stream function $\bar{f}(\eta)$, Fig. 7 for horizontal velocity $\bar{f}^{\prime}(\eta)$ and Fig. 8 for shear-stress $\bar{f}^{\prime \prime}(\eta)$. Thus, for the flow with blowing / suction, the stream function $\bar{f}(\eta)$ and horizontal velocity $\bar{f}^{\prime}(\eta)$ decrease with an increase of the slip parameter $\lambda$. Hence, we conclude that the shear-stress function $\bar{f}^{\prime \prime}(\eta)$ increases with an increase of the slip parameter $\lambda$, for all the values of the suction/blowing parameter $S$.
Also, from Fig. 9 we can notice that the variation of the concentration $\bar{\varphi}(\eta)$ decreases with the increasing of the reaction rate parameter $\beta$.

From the Tables 2 - 10 and Figs. 2 - 9 we can summarize that the approximate solutions obtained by means of the OHAM technique are effective and very accurate. This comparisons proved the accuracy, validity and flexibility of the Optimal Homotopy Asymptotic Method.

\section{Remarks:}

a) From Fluid Mechanics [30] it is known that the stream function decreases with respect to the increase of the slip parameter $\lambda$ (this signifies the loss of energy). Following most studies in this area, there exists a correlation between the loss coefficient $\lambda$ (corresponding to the slip parameter), slowing of the velocity $\bar{f}^{\prime}$ and the stream function $\bar{f}$, with the increases of the energy losses. The obtained data in the present paper correspond to the real situation from hydraulic machines (turbines, pumps, naval propellers), where the efficiency energy of the machinery is affected by the level of turbulence.

b) In the case of the hydraulic oil with a large application at the hydraulic drive systems, the analytical results obtained in our paper (using the specific physical sizes), are presented in Figs. 10 - 13.

\section{Conclusions}

In aerospace, hydraulic processes occur very often, with strongly nonlinear behaviors and even situations with singularities. Therefore, a numerical solution can capture all 
such situations and an approximate analytical solution is a more realistic option. The OHAM method does not depend upon small parameters and provides us with a convenient way to optimally control the convergence of the approximate solutions.

The analytical treatment related to the chemically reactive solute transfer problem with partial slip in the flow of a viscous fluid over an exponentially stretching sheet with suction/blowing is presented. The governing nonlinear partial differential equations (for the mass transfer and concentration) are reduced to nonlinear ordinary differential equations using some similarity transformations. The obtained nonlinear ordinary differential equations are analytically solved using the OHAM method. Some numerical examples are given for different values of the suction/blowing coefficient $S$. In the case of the flow with blowing $(S<0)$ and suction $(S>0)$, the effects of the Schmidt parameter $S c$ on the concentration $\varphi(\eta)$ are studied. The quality of the approximate solutions are made by means of the two important statistical tests: the Bartlett test and the Durbin-Wattson test. Also, the Akaike Informational Criterion (AIC) is used to make an optimal expression of the analytical solution (from our best knowledge, this tool was not used for this reason) and this simplifies the form of the solution, being easiest in physical applications.

The obtained analytical results are compared with the corresponding numerical results obtained using the fourth-order explicit Runge-Kutta method in comparison with the shooting method. The validity, flexibility, accuracy and convergence of the approximate solutions are demonstrated by means of the auxiliary functions $H_{k}\left(\eta, C_{i}\right)$ and $H_{k}^{*}\left(\eta, D_{j}\right)$, which involves the presence of the some convergence-control parameters $C_{i}$ and $D_{j}$ that are optimally identified by means of the least-squares method using the Wolfram Mathematica software 6.0.

These comparisons proved that the OHAM technique is effective and practical.

\section{Conflict of interests}

The authors declare that there is no conflict of interests regarding the publication of this paper.

\section{References}

[1] Aviation Maintenance Technician Handbook-Airframe (FAA-H8083-31) United States Department of Transportation, Federal Aviation Administration, Airman Testing Standards Branch, AFS-630, P.O. Box 25082, Oklahoma City, OK 73125.
[2] Lomen D. O., Islas A. L., Fan X., Warrick A. W., A perturbation solution for nonlinear solute transport in porous media, Transport Porous Med., 1991, 6, 739-744.

[3] Zhao C., Hobbs B. E., Ord A., Theoretical analyses of the effects of solute dispersion on chemical-dissolution front instability in fluid-saturated porous media, Transport Porous Med., 2010, 84, 629-653.

[4] Bhattacharyya K., Layek G. C., Magnetohydrodynamic slip flow and diffusion of a reactive solute past a permeable flat plate with suction/injection, Front. Chem. Sci. Eng., 2011, 5(4), 471476.

[5] Bhattacharyya K., Reactive solute transfer in a stagnation-point flow over a shrinking sheet with a diffusive mass flux, J. Appl. Mech. Tech. Phy., 2015, 56(3), 464-470.

[6] Mukhopadhyay S., Chemically reactive solute transfer in a boundary layer slip flow along a stretching cylinder, Front. Chem. Sci. Eng., 2011, 5(3), 385-391.

[7] Mukhopadhyay S., Vajravelu K., Diffusion of chemically reactive species in Casson fluid flow over an unsteady permeable stretching surface, J. Hydrodyn., 2013, 25(4), 591-598.

[8] Mukhopadhyay S., Vajravelu K., van Gorder R. A., Chemically reactive solute transfer in a moving fluid over a moving surface, Acta Mech., 2013, 224, 513-523.

[9] Mukhopadhyay S., Golam Arif M., Wazed Ali M., Effects of partial slip on chemically reactive solute transfer in the boundary layer flow over an exponentially stretching sheet with suction/blowing, J. Appl. Mech. Tech. Phy., 2013, 54(6), 928-936.

[10] Mukhopadhyay S., Chemically reactive solute transfer in boundary layer flow along a stretching cylinder in porous medium, Afr. Mat., 2014, 25, 1-10.

[11] Liao S., Beyond Perturbation: Introduction to the Homotopy Analysis Method, Chapman and Hall, 2003.

[12] Marinca V., Herisanu N., The Optimal Homotopy Asymptotic Method - Engineering Applications, Springer Verlag, Heidelberg, 2015.

[13] Marinca V., Ene R.-D., Marinca B., Negrea R., Different approximations to the solution of upper-convected Maxwell fluid over a porous stretching plate, Abstr. Appl. Anal., 2014, Article ID 139314, 13 pages.

[14] Ene R.-D., Marinca V., Approximate solutions for steady boundary layer MHD viscous flow and radiative heat transfer over an exponentially porous stretching sheet, Appl. Math. Comput., 2015, 269, 389-401.

[15] Ene R.-D., Szabo M. A., Danoiu S., Viscous flow and heat transfer over a permeable shrinking sheet with partial slip, Mater. Plast., 2015, 52(3), 408-412.

[16] Marinca V., Ene R.-D., Dual approximate solutions of the unsteady viscous flow over a shrinking cylinder with Optimal Homotopy Asymptotic Method, Adv. Math. Phys., 2014, Article ID 417643, 11 pages.

[17] Li S., Karatzoglu A., Gentile C., Collaborative Filtering Bandits, Proceedings of the 39th International ACM SIGIR Conference on Research and Development in Information Retrieval, (SIGIR 2016, Pisa, Tuscany, Italy), 539-548.

[18] Gentile C., Li S., et al., On Context-Dependent Clustering of Bandits, Proceedings of the 34th International Conference on Machine Learning, (ICML 2017, Sydney, Australia), J. Mach. Learn. Res., 2017, 1253-1262.

[19] Kar P., Li S., et al., Online Optimization Methods for the Quantification Problem, Proceedings of the 22nd ACM SIGKDD Inter- 
national Conference on Knowledge Discovery and Data Mining, (SIGKDD 2016, San Francisco, California, US), 1625-1634.

[20] Hao F., Park D.-S., Li S., Lee H. M., Mining $\lambda$-Maximal Cliques from a Fuzzy Graph, Journal of Sustainability, 2016, 8(6), 2016, pp. 553.

[21] Li S., The Art of Clustering Bandits, M.Sc. thesis, Universita degli Studi dell'Insubria, 2016.

[22] Hao F., Li S., et al., An Efficient Approach to Generating LocationSensitive Recommendations in Ad-hoc Social Network Environments, IEEE T. Serv. Comput., 2015, 99, 520-533.

[23] Gentile C., Li S., Zappella G., Online Clustering of Bandits, Proceedings of the 31st International Conference on Machine Learning, (ICML 2014, Beijing, China), J. Mach. Learn. Res., 2014, 757-765.

[24] Li S., et al., Medicine Rating Prediction and Recommendation in Mobile Social Networks, Proceedings of the International Conference on Green and Pervasive Computing, (May 9-11, 2013, Seoul, Korea), 2013, Vol. 7861, 216-223.

[25] Guo H., Feng Yi, et al., Dynamic Fuzzy Logic Control of Genetic Algorithm Probabilities, Journal of Computers, 2014, 9(1), 22-27.

[26] Korda N., Szorenyi B., Distributed Clustering of Linear Bandits in Peer to Peer Networks, Proceedings of the 33rd International Conference on Machine Learning, (ICML 2016, New York City, NY, US), J. Mach. Learn. Res., 2016, 1301-1309.

[27] Martin M. J., Boyd I. D., Momentum and heat transfer in a laminar boundary layer with slip flow, J. Thermo. Heat Trans., 2006, 20(4), 710-719.

[28] Anderson H. I., Slip flow past a stretching surface, Acta Mech., 2002, 158, 121-125.

[29] Akaike H., A new look at the statistical model identification, IEEE Transaction on Automatic Control, 1974, 19(6), 716-723.

[30] Giles Ranald V., Theory and problems of the Hydraulics $2^{\text {nd }}$ ed., Schaum's Outline Series, McGraw Hill Book Company, 1977.

\section{Appendix}

$\mathbf{a}_{2}$ ) the reaction rate parameter $\beta=0.1$, the Schmidt parameter $S c=2$.

$$
\begin{gathered}
\bar{\varphi}(\eta)=-\frac{4521.9604188190}{(1+0.4805800000 \eta)^{4}}+ \\
+\frac{2356.0603762273}{(1+0.4805800000 \eta)^{3}}-\frac{847.9472565809}{(1+0.4805800000 \eta)^{2}}+ \\
+\frac{255.6310251627}{1+0.4805800000 \eta}+[-11.5977491489- \\
-\frac{1581.9630677767}{(1+0.4805800000 \eta)^{10}}+\frac{6422.8119168821}{(1+0.4805800000 \eta)^{9}}+ \\
\left.+\frac{75.4026094077 \eta^{2}}{(1+0.4805800000 \eta)^{11}}\right] \cdot \frac{1}{(1+0.0823625270 \eta)^{2}}+ \\
+\left[-43.7890004039-\frac{9655.6113912982}{(1+0.4805800000 \eta)^{7}}-\right. \\
-\frac{1381.6997787255}{(1+0.4805800000 \eta)^{6}}+\frac{9011.0653444810}{(1+0.4805800000 \eta)^{5}}-
\end{gathered}
$$

$$
\left.-\frac{3288.3113357558 \eta^{2}}{(1+0.4805800000 \eta)^{8}}\right] \cdot \frac{1}{1+0.0823625270 \eta} .
$$

$\mathbf{a}_{3}$ ) the reaction rate parameter $\beta=0.1$, the Schmidt parameter $S c=3$.

$$
\begin{gathered}
\bar{\varphi}(\eta)=-\frac{319.4742713350}{(1+0.3927894125 \eta)^{4}}+ \\
+\frac{318.1469012244}{(1+0.3927894125 \eta)^{3}}-\frac{181.2395203884}{(1+0.3927894125 \eta)^{2}}+ \\
+\frac{78.27140387039}{1+0.3927894125 \eta}+[-5.2068252742- \\
-\frac{73.6271291945}{(1+0.3927894125 \eta)^{10}}+\frac{11.8165939907}{(1+0.3927894125 \eta)^{9}}+ \\
\left.+\frac{9.3644276148 \eta^{2}}{(1+0.3927894125 \eta)^{11}}\right] \cdot \frac{1}{(1+0.0823625270 \eta)^{2}}+ \\
+\left[-16.3889604583+\frac{81.1888081932}{(1+0.3927894125 \eta)^{7}}+\right. \\
+\frac{126.7002988542}{(1+0.3927894125 \eta)^{6}}-\frac{19.1872994825}{(1+0.3927894125 \eta)^{5}}+ \\
\left.+\frac{88.4672480728 \eta^{2}}{(1+0.3927894125 \eta)^{8}}\right] \cdot \frac{1}{1+0.0823625270 \eta} .
\end{gathered}
$$

$\mathbf{a}_{4}$ ) the reaction rate parameter $\beta=0.1$, the Schmidt parameter $S c=10$.

$$
\begin{gathered}
\bar{\varphi}(\eta)=\frac{6672.6048712372}{(1+0.7905481787 \eta)^{4}}- \\
-\frac{2311.9203057744}{(1+0.7905481787 \eta)^{3}}+\frac{531.5964075112}{(1+0.7905481787 \eta)^{2}}- \\
-\frac{97.8239775577}{1+0.7905481787 \eta}+[3.5234687767+ \\
+\frac{7444.1818776794}{(1+0.7905481787 \eta)^{10}}-\frac{28233.4084196663}{(1+0.7905481787 \eta)^{9}}- \\
\left.-\frac{1075.2439347959 \eta^{2}}{(1+0.7905481787 \eta)^{11}}\right] \cdot \frac{1}{(1+0.0823625270 \eta)^{2}}+ \\
+\left[10.1731902044+\frac{47233.6747898730}{(1+0.7905481787 \eta)^{7}}-\right. \\
-\frac{12771.8642842899}{(1+0.7905481787 \eta)^{6}}-\frac{18479.7376179937}{(1+0.7905481787 \eta)^{5}}+ \\
\left.+\frac{32753.0809355307 \eta^{2}}{(1+0.7905481787 \eta)^{8}}\right] \cdot \frac{1}{1+0.0823625270 \eta} .
\end{gathered}
$$

Example 5.2 If we consider $S=0, \lambda=0.1$, the expression of the first-order approximate solution is:

$$
\bar{f}(\eta)=0.8553480468+\frac{6.9711159079}{(1+0.1566842159 \eta)^{11}}-
$$


Table 3: Comparison between OHAM results $\left(\bar{f}, \bar{f}^{\prime}\right)$ given by Eq. (49) and numerical results, for $S=0, \lambda=0.1$ (relative errors: $\epsilon_{f}=\left|f_{\text {numerical }}-\bar{f}_{\text {OHAM }}\right|, \epsilon_{f^{\prime}}=\left|f_{\text {numerical }}^{\prime}-\bar{f}_{\text {OHAM }}^{\prime}\right|$ )

\begin{tabular}{lllllll}
\hline$\eta$ & $f_{\text {numerical }}$ & $\begin{array}{l}\bar{f}_{\text {OHAM }} \\
\text { given by } \\
\text { Eq. (49) }\end{array}$ & $\epsilon_{f}$ & $f_{\text {numerical }}^{\prime}$ & $\begin{array}{l}\bar{f}_{\text {OHAM }}^{\prime} \\
\text { from } \\
\text { Eq. (49) }\end{array}$ & $\epsilon_{f^{\prime}}$ \\
\hline 0 & 0 & 0 & 0 & 0.8920112191 & 0.8920112291 & $1.00 \cdot 10^{-8}$ \\
$4 / 5$ & 0.4673897518 & 0.4673537169 & $3.60 \cdot 10^{-5}$ & 0.3670253355 & 0.3670394113 & $1.40 \cdot 10^{-5}$ \\
$8 / 5$ & 0.6695649982 & 0.6695830109 & $1.80 \cdot 10^{-5}$ & 0.1672482217 & 0.1673093023 & $6.10 \cdot 10^{-5}$ \\
$12 / 5$ & 0.7640237918 & 0.7640465048 & $2.27 \cdot 10^{-5}$ & 0.0801628206 & 0.0801224649 & $4.03 \cdot 10^{-5}$ \\
$16 / 5$ & 0.8098725930 & 0.8098550411 & $1.75 \cdot 10^{-5}$ & 0.0394093404 & 0.0393649182 & $4.44 \cdot 10^{-5}$ \\
$20 / 5$ & 0.8325566117 & 0.8325209434 & $3.56 \cdot 10^{-5}$ & 0.0196234072 & 0.0196233607 & $4.64 \cdot 10^{-6}$ \\
$24 / 5$ & 0.8438882353 & 0.8438656573 & $2.25 \cdot 10^{-5}$ & 0.0098344018 & 0.0098622420 & $2.78 \cdot 10^{-5}$ \\
$28 / 5$ & 0.8495763831 & 0.8495777875 & $1.40 \cdot 10^{-6}$ & 0.0049446204 & 0.0049730441 & $2.84 \cdot 10^{-5}$ \\
$32 / 5$ & 0.8524386621 & 0.8524577684 & $1.91 \cdot 10^{-5}$ & 0.0024901777 & 0.0025048868 & $1.47 \cdot 10^{-5}$ \\
$36 / 5$ & 0.8538807397 & 0.8539053805 & $2.46 \cdot 10^{-5}$ & 0.0012551227 & 0.0012547545 & $3.68 \cdot 10^{-7}$ \\
$40 / 5$ & 0.8546077411 & 0.8546276346 & $1.98 \cdot 10^{-5}$ & 0.0006328835 & 0.0006224246 & $1.04 \cdot 10^{-5}$ \\
\hline & & & & & & \\
\hline
\end{tabular}

$$
\begin{gathered}
-\frac{23.4064493240}{(1+0.1566842159 \eta)^{10}}+\frac{18.3815081245}{(1+0.1566842159 \eta)^{9}}+ \\
+\frac{17.8208421539}{(1+0.1566842159 \eta)^{8}}-\frac{32.4126426760}{(1+0.1566842159 \eta)^{7}}+ \\
+\frac{8.4627611959}{(1+0.1566842159 \eta)^{6}}+\frac{7.6283127417}{(1+0.1566842159 \eta)^{5}}- \\
-\frac{5.5911050001}{(1+0.1566842159 \eta)^{4}}+\frac{1.4500589220}{(1+0.1566842159 \eta)^{3}}- \\
-\frac{0.1667819023}{(1+0.1566842159 \eta)^{2}}+\frac{0.0070318095}{1+0.1566842159 \eta}- \\
-\frac{0.0082360507 \eta^{2}}{(1+0.1566842159 \eta)^{14}} .
\end{gathered}
$$

Example 5.3 In the last case, we consider $S=0.5$, $\lambda=0.1$. For Eq. (31), the first-order approximate solution becomes:

$$
\begin{gathered}
\bar{f}(\eta)=0.3951796613-\frac{3271.6197302461}{(1+0.1557538731 \eta)^{11}}+ \\
+\frac{16449.3646199342}{(1+0.1557538731 \eta)^{10}}-\frac{35947.1857764394}{(1+0.1557538731 \eta)^{9}}+ \\
+\frac{44518.5700211918}{(1+0.1557538731 \eta)^{8}}-\frac{34063.9357494410}{(1+0.1557538731 \eta)^{7}}+ \\
+\frac{16498.6618355929}{(1+0.1557538731 \eta)^{6}}-\frac{5041.9301562207}{(1+0.1557538731 \eta)^{5}}+ \\
\frac{962.0040557256}{(1+0.1557538731 \eta)^{4}}-\frac{110.6177176818}{(1+0.1557538731 \eta)^{3}}+ \\
+\frac{6.9779181485}{(1+0.1557538731 \eta)^{2}}-\frac{0.1845002254}{1+0.1557538731 \eta}+ \\
+\frac{2.6642660182 \eta^{2}}{(1+0.1557538731 \eta)^{14}}
\end{gathered}
$$

In what follows, we present an analytical study of the effect of the partial slip parameter $\lambda$ on the chemically reactive solute transfer. In this way, we give the approximate analytical solutions for the cases: $S=-0.5<0$ - flow with blowing, $S=0$ and $S=0.5>0$ - flow with suction.

Example 5.4 If we consider $S=-0.5, \lambda=0.3$, for Eq. (31), the first-order approximate solution becomes:

$$
\begin{gathered}
\bar{f}(\eta)=0.5411801898+\frac{7.3478010166}{(1+0.1085405812 \eta)^{11}}- \\
-\frac{17.9394900127}{(1+0.1085405812 \eta)^{10}}+\frac{1.5448818611}{(1+0.1085405812 \eta)^{9}}+ \\
+\frac{22.2186265177}{(1+0.1085405812 \eta)^{8}}-\frac{1.6369226318}{(1+0.1085405812 \eta)^{7}}- \\
-\frac{34.5645028758}{(1+0.1085405812 \eta)^{6}}+\frac{32.7905087261}{(1+0.1085405812 \eta)^{5}}- \\
-\frac{13.2364284405}{(1+0.1085405812 \eta)^{4}}+\frac{2.6915291658}{(1+0.1085405812 \eta)^{3}}- \\
-\frac{0.2673717709}{(1+0.1085405812 \eta)^{2}}+\frac{0.0101882545}{1+0.1085405812 \eta}- \\
-\frac{0.0197293858 \eta^{2}}{(1+0.1085405812 \eta)^{14}} .
\end{gathered}
$$

Example 5.5 In the case when $S=-0.5, \lambda=0.5$, the first-order approximate solution Eq. (31) becomes:

$$
\begin{gathered}
\bar{f}(\eta)=0.4984555713+\frac{2.237489771485}{(1+0.137707597908 \eta)^{11}}- \\
-\frac{2.3944957680}{(1+0.137707597908 \eta)^{10}}-\frac{3.3763171999}{(1+0.137707597908 \eta)^{9}}+ \\
+\frac{0.0246528401}{(1+0.137707597908 \eta)^{8}}+\frac{6.5905945484}{(1+0.137707597908 \eta)^{7}}+
\end{gathered}
$$


Table 4: Comparison between OHAM results $\left(\bar{f}, \bar{f}^{\prime}\right)$ given by Eq. (50) and numerical results, for $S=0.5, \lambda=0.1$ (relative errors: $\epsilon_{f}=\left|f_{\text {numerical }}-\bar{f}_{\text {OHAM }}\right|, \epsilon_{f^{\prime}}=\left|f_{\text {numerical }}^{\prime}-\bar{f}_{\text {OHAM }}^{\prime}\right|$ )

\begin{tabular}{lllllll}
\hline$\eta$ & $f_{\text {numerical }}$ & $\begin{array}{l}\bar{f}_{\text {OHAM }} \\
\text { given by } \\
\text { Eq. (50) }\end{array}$ & $\epsilon_{f}$ & $f_{\text {numerical }}^{\prime}$ & $\begin{array}{l}\bar{f}_{\text {OHAM }}^{\prime} \\
\text { from } \\
\text { Eq. (50) }\end{array}$ & $\epsilon_{f^{\prime}}$ \\
\hline 0 & 0.5 & 0.5 & 0 & 0.8688355156 & 0.8688355206 & $4.96 \cdot 10^{-9}$ \\
$4 / 5$ & 0.9053218753 & 0.9053254228 & $3.54 \cdot 10^{-6}$ & 0.2582388571 & 0.2583911834 & $1.52 \cdot 10^{-4}$ \\
$8 / 5$ & 1.0103156474 & 1.0103053782 & $1.02 \cdot 10^{-5}$ & 0.0365073971 & 0.0363862320 & $1.21 \cdot 10^{-4}$ \\
$12 / 5$ & 0.9961189516 & 0.9960895510 & $2.94 \cdot 10^{-5}$ & -0.0590709734 & -0.0589898768 & $8.10 \cdot 10^{-5}$ \\
$16 / 5$ & 0.9304509566 & 0.9304955172 & $4.45 \cdot 10^{-5}$ & -0.0985676220 & -0.0985102893 & $5.73 \cdot 10^{-5}$ \\
$20 / 5$ & 0.8461894701 & 0.8462286423 & $3.91 \cdot 10^{-5}$ & -0.1085349302 & -0.1085936980 & $5.87 \cdot 10^{-5}$ \\
$24 / 5$ & 0.7609466483 & 0.7609297394 & $1.69 \cdot 10^{-5}$ & -0.1027674406 & -0.1028300572 & $6.26 \cdot 10^{-5}$ \\
$28 / 5$ & 0.6836993747 & 0.6836553875 & $4.39 \cdot 10^{-5}$ & -0.0896263299 & -0.0896290671 & $2.73 \cdot 10^{-6}$ \\
$32 / 5$ & 0.6181395908 & 0.6181131443 & $2.64 \cdot 10^{-5}$ & -0.0741793261 & -0.0741391579 & $4.01 \cdot 10^{-5}$ \\
$36 / 5$ & 0.5648641651 & 0.5648736781 & $9.51 \cdot 10^{-6}$ & -0.0592394367 & -0.0591955250 & $4.39 \cdot 10^{-5}$ \\
$40 / 5$ & 0.5228604069 & 0.5228977468 & $3.73 \cdot 10^{-5}$ & -0.0461306368 & -0.0461071944 & $2.34 \cdot 10^{-5}$ \\
\hline & & & & & &
\end{tabular}

$$
\begin{aligned}
& +\frac{5.5468929502}{(1+0.137707597908 \eta)^{6}}-\frac{19.1304248662}{(1+0.137707597908 \eta)^{5}}+ \\
& +\frac{12.5851210119}{(1+0.137707597908 \eta)^{4}}-\frac{3.5027615145}{(1+0.137707597908 \eta)^{3}}+
\end{aligned}
$$$$
+\frac{0.4412703528}{(1+0.137707597908 \eta)^{2}}-\frac{0.0204776978}{1+0.1377075979 \eta}-
$$

$$
-\frac{0.0206505631 \eta^{2}}{(1+0.1377075979 \eta)^{14}} \text {. }
$$

The influence of the reaction rate parameter $\beta$ on the chemically reactive solute transfer is presented below. In this way we give the approximate analytical solutions for the cases: $\beta=0.1, \beta=0.3$ and $\beta=1$, where $S=-0.5, \lambda=0.1$ and $S c=0.7$ are fixed.

Example 5.6 In the first case, we consider $S=-0.5$, $\lambda=0.1, S c=0.7$ and $\beta=0.1$. For Eq. (41), the first-order approximate solution becomes:

$$
\begin{gathered}
\bar{\varphi}(\eta)=-\frac{3.9816029319}{(1+0.1646305546 \eta)^{4}}+ \\
+\frac{4.0335307886}{(1+0.1646305546 \eta)^{3}}-\frac{0.1534745401}{(1+0.1646305546 \eta)^{2}}- \\
-\frac{5.0593327058}{1+0.1646305546 \eta}+[1.3512198945- \\
-\frac{3.1027133726}{(1+0.1646305546 \eta)^{10}}+\frac{1.3429468640}{(1+0.1646305546 \eta)^{9}}+ \\
\left.\left.+\frac{0.0671762598 \eta^{2}}{(1+0.1646305546 \eta)^{11}}\right)\right] \cdot \frac{1}{(1+0.0823625270 \eta)^{2}}+ \\
+\left[2.5267225444+\frac{2.9456463231}{(1+0.1646305546 \eta)^{7}}+\right. \\
+\frac{2.9607435234}{(1+0.1646305546 \eta)^{6}}-\frac{1.8636863877}{(1+0.1646305546 \eta)^{5}}+
\end{gathered}
$$

$$
\left.+\frac{0.6559223088 \eta^{2}}{(1+0.1646305546 \eta)^{8}}\right] \cdot \frac{1}{1+0.0823625270 \eta} .
$$

Example 5.7 In the second case, if $S=-0.5, \lambda=0.1$, $S c=0.7$ and $\beta=0.3$, the first-order approximate solution Eq. (41) becomes:

$$
\begin{gathered}
\bar{\varphi}(\eta)=-\frac{18.5366044376}{(1+0.1829601791 \eta)^{4}}+ \\
+\frac{9.8353575515}{(1+0.1829601791 \eta)^{3}}+\frac{9.3313946862}{(1+0.1829601791 \eta)^{2}}- \\
-\frac{25.0062589622}{1+0.1829601791 \eta}+[4.4116732762- \\
-\frac{7.3153836234}{(1+0.1829601791 \eta)^{10}}+\frac{1.4081543441}{(1+0.1829601791 \eta)^{9}}+ \\
\left.+\frac{0.2101153153 \eta^{2}}{(1+0.1829601791 \eta)^{11}}\right] \cdot \frac{1}{(1+0.0823625270 \eta)^{2}}+ \\
+\left[11.2464497840+\frac{7.8589046140}{(1+0.1829601791 \eta)^{7}}+\right. \\
+\frac{10.8712439005}{(1+0.1829601791 \eta)^{6}}-\frac{3.1049311335}{(1+0.1829601791 \eta)^{5}}+ \\
\left.+\frac{2.2057006407 \eta^{2}}{(1+0.1829601791 \eta)^{8}}\right] \cdot \frac{1}{1+0.0823625270 \eta} .
\end{gathered}
$$

Example 5.8 In the last case, we consider $S=0$, $\lambda=0.1, S c=0.7$ and $\beta=1$. The first-order approximate solution given by Eq. (41) becomes:

$$
\bar{\varphi}(\eta)=-\frac{11.3571514687}{(1+0.1303333753 \eta)^{4}}+
$$


Table 5: Comparison between the approximate solutions $\varphi$ given by Eqs. (43), (46), (47), (48) and numerical results for $\lambda=0.1, S=-0.5$, $\beta=0.1$ and different values of the Schmidt parameter $S c$ (relative errors: $\epsilon_{\varphi}=\left|\varphi_{\text {numerical }}-\bar{\varphi}_{\text {OHAM }}\right|$ )

\begin{tabular}{|c|c|c|c|c|}
\hline & $S c=0.7$ & $S c=2$ & $S c=3$ & $S c=10$ \\
\hline$\eta$ & $\varphi_{\text {numerical }}$ & $\varphi_{\text {numerical }}$ & $\varphi_{\text {numerical }}$ & $\varphi_{\text {numerical }}$ \\
\hline 0 & 1 & 1 & 1 & 1 \\
\hline $7 / 10$ & 0.6563309653 & 0.4811705634 & 0.4133356959 & 0.2407707922 \\
\hline $7 / 5$ & 0.4413426637 & 0.2217337394 & 0.1525057373 & 0.0288952290 \\
\hline $21 / 10$ & 0.3013144063 & 0.0982608275 & 0.0509589853 & 0.0017932391 \\
\hline $14 / 5$ & 0.2081737030 & 0.0424443546 & 0.0158971840 & 0.0000668552 \\
\hline $7 / 2$ & 0.1453929006 & 0.0181266146 & 0.0047700395 & $3.7073158658 \cdot 10^{-6}$ \\
\hline $21 / 5$ & 0.1026272704 & 0.0077469672 & 0.0014107659 & $4.8504497218 \cdot 10^{-7}$ \\
\hline $49 / 10$ & 0.0732049286 & 0.0033449264 & 0.0004190735 & $-2.3227934713 \cdot 10^{-6}$ \\
\hline $28 / 5$ & 0.0527572988 & 0.0014693029 & 0.0001267826 & $1.1093472718 \cdot 10^{-6}$ \\
\hline $63 / 10$ & 0.0383982655 & 0.0006597352 & 0.0000394466 & $2.4540273639 \cdot 10^{-6}$ \\
\hline 7 & 0.0282077046 & 0.0003036887 & 0.0000127039 & $-5.2390950688 \cdot 10^{-8}$ \\
\hline$\eta$ & $\begin{array}{l}\bar{\varphi}_{O H A M} \\
\text { given by Eq. (43) }\end{array}$ & $\begin{array}{l}\bar{\varphi}_{O H A M} \\
\text { given by Eq. (46) }\end{array}$ & $\begin{array}{l}\bar{\varphi}_{O H A M} \\
\text { given by Eq. (47) }\end{array}$ & $\begin{array}{l}\bar{\varphi}_{O H A M} \\
\text { given by Eq. (48) }\end{array}$ \\
\hline 0 & 1 & 1 & 1 & 1 \\
\hline $7 / 10$ & 0.6563234894 & 0.4811687367 & 0.4133697770 & 0.2407413591 \\
\hline $7 / 5$ & 0.4413508444 & 0.2217397226 & 0.1524582990 & 0.0290780437 \\
\hline $21 / 10$ & 0.3013056724 & 0.0982524310 & 0.0510094743 & 0.0015675514 \\
\hline $14 / 5$ & 0.2081583194 & 0.0424480615 & 0.0159244709 & 0.0001859219 \\
\hline $7 / 2$ & 0.1453900629 & 0.0181339184 & 0.0047225861 & 0.0001553578 \\
\hline $21 / 5$ & 0.1026386818 & 0.0077446788 & 0.0013713877 & -0.0000195483 \\
\hline $49 / 10$ & 0.0732206957 & 0.0033377499 & 0.0004241938 & -0.0001091419 \\
\hline $28 / 5$ & 0.0527674614 & 0.0014651713 & 0.0001581552 & -0.0000923121 \\
\hline $63 / 10$ & 0.0383983366 & 0.0006609783 & 0.0000683943 & -0.0000307619 \\
\hline 7 & 0.0281986506 & 0.0003081183 & 0.0000230022 & 0.0000280374 \\
\hline$\eta$ & $\begin{array}{l}\epsilon_{\varphi} \\
\text { for Eq. (43) }\end{array}$ & $\begin{array}{l}\epsilon_{\varphi} \\
\text { for Eq. (46) }\end{array}$ & $\begin{array}{l}\epsilon_{\varphi} \\
\text { for Eq. (47) }\end{array}$ & $\begin{array}{l}\epsilon_{\varphi} \\
\text { for Eq. (48) }\end{array}$ \\
\hline 0 & 0 & 0 & 0 & 0 \\
\hline $7 / 10$ & $7.47 \cdot 10^{-6}$ & $1.82 \cdot 10^{-6}$ & $3.40 \cdot 10^{-5}$ & $2.94 \cdot 10^{-5}$ \\
\hline $7 / 5$ & $8.18 \cdot 10^{-6}$ & $5.98 \cdot 10^{-6}$ & $4.74 \cdot 10^{-5}$ & $1.82 \cdot 10^{-4}$ \\
\hline $21 / 10$ & $8.73 \cdot 10^{-6}$ & $8.39 \cdot 10^{-6}$ & $5.04 \cdot 10^{-5}$ & $2.25 \cdot 10^{-4}$ \\
\hline $14 / 5$ & $1.53 \cdot 10^{-5}$ & $3.70 \cdot 10^{-6}$ & $2.72 \cdot 10^{-5}$ & $1.19 \cdot 10^{-4}$ \\
\hline $7 / 2$ & $2.83 \cdot 10^{-6}$ & $7.30 \cdot 10^{-6}$ & $4.74 \cdot 10^{-5}$ & $1.51 \cdot 10^{-4}$ \\
\hline $21 / 5$ & $1.14 \cdot 10^{-5}$ & $2.28 \cdot 10^{-6}$ & $3.93 \cdot 10^{-5}$ & $2.003 \cdot 10^{-5}$ \\
\hline $49 / 10$ & $1.57 \cdot 10^{-5}$ & $7.17 \cdot 10^{-6}$ & $5.12 \cdot 10^{-6}$ & $1.06 \cdot 10^{-4}$ \\
\hline $28 / 5$ & $1.01 \cdot 10^{-5}$ & $4.13 \cdot 10^{-6}$ & $3.13 \cdot 10^{-5}$ & $9.34 \cdot 10^{-5}$ \\
\hline $63 / 10$ & $7.10 \cdot 10^{-8}$ & $1.24 \cdot 10^{-6}$ & $2.89 \cdot 10^{-5}$ & $3.32 \cdot 10^{-5}$ \\
\hline 7 & $9.05 \cdot 10^{-6}$ & $4.42 \cdot 10^{-6}$ & $1.02 \cdot 10^{-5}$ & $2.80 \cdot 10^{-5}$ \\
\hline
\end{tabular}

$$
\begin{gathered}
+\frac{15.1673641129}{(1+0.1303333753 \eta)^{3}}+\frac{9.8211516277}{(1+0.1303333753 \eta)^{2}}- \\
-\frac{87.2816410939}{1+0.1303333753 \eta}+[16.4331024534-
\end{gathered}
$$$$
-\frac{5.4788082482}{(1+0.1303333753 \eta)^{10}}+\frac{3.3996212268}{(1+0.1303333753 \eta)^{9}}+
$$$$
\left.+\frac{0.1624234907 \eta^{2}}{(1+0.1303333753 \eta)^{11}}\right] \cdot \frac{1}{(1+0.0823625270 \eta)^{2}}+
$$ 


$$
\begin{gathered}
+\left[55.1840641665+\frac{6.7234323305}{(1+0.1303333753 \eta)^{7}}+\right. \\
+\frac{4.1693318998}{(1+0.1303333753 \eta)^{6}}-\frac{5.7804670069}{(1+0.1303333753 \eta)^{5}}+ \\
\left.+\frac{0.3600618455 \eta^{2}}{(1+0.1303333753 \eta)^{8}} \cdot\right] \frac{1}{1+0.0823625270 \eta} .
\end{gathered}
$$

This way, we can construct other accurate approximate solutions.

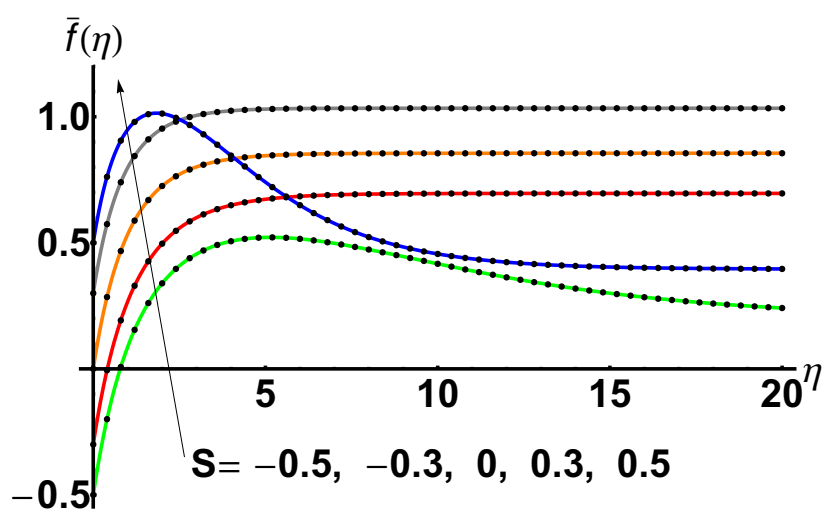

Figure 2: Variation of the stream function $\bar{f}(\eta)$ with increasing of the suction/blowing parameter $S$ for $\lambda=0.1$ :

— OHAM solution ; $\bullet \cdots \bullet \bullet \bullet$ numerical solution

Table 6: Comparison between the skin-friction coefficient $\bar{f}^{\prime \prime}(0)$ obtained by means of the OHAM and numerical results, for $\lambda=0.1$ and different values of the suction/blowing parameter $S$ (relative errors: $\left.\epsilon_{f^{\prime \prime}(0)}=\left|f_{\text {numerical }}^{\prime \prime}(0)-\bar{f}_{O H A M}^{\prime \prime}(0)\right|\right)$

\begin{tabular}{llll}
\hline$S$ & $f_{\text {numerical }}^{\prime \prime}(0)$ & $\bar{f}_{\text {OHAM }}^{\prime \prime}(0)$ & $\epsilon_{f^{\prime \prime}(0)}$ \\
\hline-0.5 & -0.9222268732 & -0.9222267721 & $1.01 \cdot 10^{-7}$ \\
-0.3 & -0.9808736678 & -0.9808736578 & $1.00 \cdot 10^{-8}$ \\
0 & -1.0798878084 & -1.0798877084 & $1.00 \cdot 10^{-7}$ \\
0.3 & -1.1913158524 & -1.1913157524 & $9.99 \cdot 10^{-8}$ \\
0.5 & -1.3116448434 & -1.3116447938 & $4.96 \cdot 10^{-8}$ \\
\hline
\end{tabular}

The obtained results are in agreement with the fluid flow scenario from a hydraulic installation for different values of the characteristic quantities: $U_{0}=0.1[\mathrm{~m} / \mathrm{s}]$, $C_{0}=0.1, v=46 \cdot 10^{-6}\left[\mathrm{~m}^{2} / \mathrm{s}\right]$ and $C_{\infty}=0.1$, respectively.
Table 7: Comparison between the limit value $\bar{f}(\infty)$ obtained by means of the OHAM and numerical results, for $\lambda=0.1$ and different values of the suction/blowing parameter $S$ (relative errors: $\left.\epsilon_{f(\infty)}=\left|f_{\text {numerical }}(\infty)-\bar{f}_{\text {OHAM }}(\infty)\right|\right)$

\begin{tabular}{llll}
\hline$S$ & $f_{\text {numerical }}(\infty)$ & $\bar{f}_{\text {OHAM }}(\infty)$ & $\epsilon_{f(\infty)}$ \\
\hline-0.5 & 0.2032088541 & 0.2032088596 & $5.54 \cdot 10^{-9}$ \\
-0.3 & 0.6960935296 & 0.6960938006 & $2.71 \cdot 10^{-7}$ \\
0 & 0.8553478033 & 0.8553480468 & $2.43 \cdot 10^{-7}$ \\
0.3 & 1.0339634884 & 1.0339644067 & $9.18 \cdot 10^{-7}$ \\
0.5 & 0.3951795612 & 0.3951796613 & $1.00 \cdot 10^{-7}$ \\
\hline
\end{tabular}

Table 8: Comparison between the mass transfer coefficient $\bar{\varphi}^{\prime}(0)$ obtained by means of the OHAM and numerical results, for $S=$ $-0.5, \lambda=0.1$ and different values of the Schmidt coefficient $S c$ (relative errors: $\epsilon_{\varphi^{\prime}(0)}=\left|\varphi_{\text {numerical }}^{\prime}(0)-\bar{\varphi}_{\text {OHAM }}^{\prime}(0)\right|$ )

\begin{tabular}{llll}
\hline$S c$ & $\varphi_{\text {numerical }}^{\prime}(0)$ & $\bar{\varphi}_{\text {OHAM }}^{\prime}(0)$ & $\epsilon_{\varphi^{\prime}(0)}$ \\
\hline 0.7 & -0.6273507907 & -0.6273506907 & $1.00 \cdot 10^{-7}$ \\
2 & -1.0206863072 & -1.0206862840 & $2.31 \cdot 10^{-8}$ \\
3 & -1.1891244067 & -1.1891243967 & $1.00 \cdot 10^{-8}$ \\
10 & -1.6436491544 & -1.6436491442 & $1.02 \cdot 10^{-8}$ \\
\hline
\end{tabular}

Table 9: Comparison between the mass transfer coefficient $\bar{\varphi}^{\prime}(0)$ obtained by means of the OHAM and numerical results, for $S=0.5$, $\lambda=0.1$ and different values of the Schmidt coefficient $S c$ (relative errors: $\left.\epsilon_{\varphi^{\prime}(0)}=\left|\varphi_{\text {numerical }}^{\prime}(0)-\bar{\varphi}_{\text {OHAM }}^{\prime}(0)\right|\right)$

\begin{tabular}{llll}
\hline$S c$ & $\varphi_{\text {numerical }}^{\prime}(0)$ & $\bar{\varphi}_{\text {OHAM }}^{\prime}(0)$ & $\epsilon_{\varphi^{\prime}(0)}$ \\
\hline 0.7 & -0.9028032434 & -0.9028031434 & $9.99 \cdot 10^{-8}$ \\
2 & -2.0537968062 & -2.0537967962 & $1.00 \cdot 10^{-8}$ \\
3 & -2.7842989909 & -2.7842989809 & $9.99 \cdot 10^{-9}$ \\
10 & -7.0577817249 & -7.0577816249 & $9.99 \cdot 10^{-8}$ \\
\hline
\end{tabular}

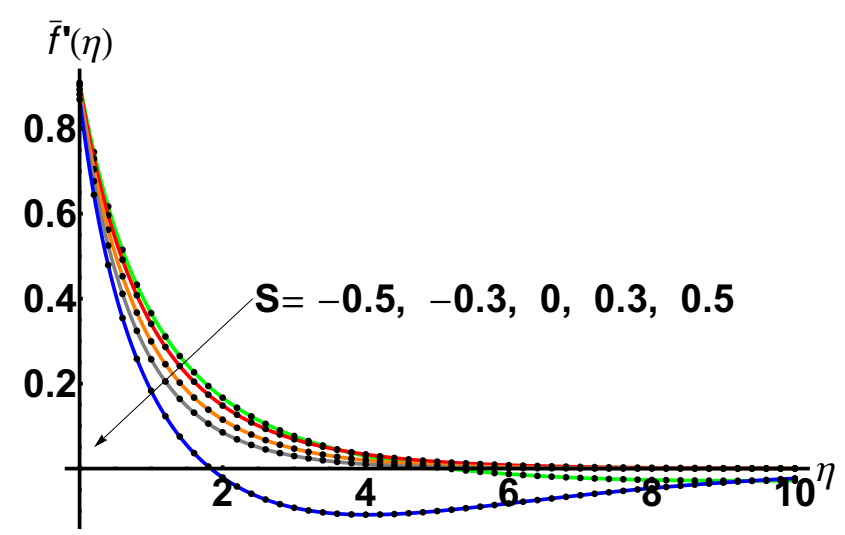

Figure 3: Variation of the horizontal velocity $\bar{f}^{\prime}(\eta)$ with increasing of the suction/blowing parameter $S$ for $\lambda=0.1$ :

- OHAM solution ; $\bullet \cdots \bullet \cdot \bullet$ numerical solution 
Table 10: Integral of the square residual given by Eqs (44) and (45) respectively, for different values of the parameters $S, \lambda, \beta$ and $S c$

$S \quad \lambda \quad \beta \quad S c \quad \int_{0}^{\infty} R_{\bar{f}}^{2}(\eta) d \eta \quad \int_{0}^{\infty} R_{\bar{\varphi}}^{2}(\eta) d \eta$

\begin{tabular}{llllll}
\hline-0.5 & 0.1 & 0.1 & 0.7 & $8.08 \cdot 10^{-6}$ & $7.94 \cdot 10^{-8}$ \\
-0.5 & 0.1 & 0.1 & 2 & & $1.25 \cdot 10^{-5}$ \\
-0.5 & 0.1 & 0.1 & 3 & & $1.45 \cdot 10^{-4}$ \\
-0.5 & 0.1 & 0.3 & 0.7 & & $2.46 \cdot 10^{-6}$ \\
-0.5 & 0.1 & 1 & 0.7 & & $9.04 \cdot 10^{-4}$ \\
\hline 0.5 & 0.1 & 0.1 & 0.7 & $4.03 \cdot 10^{-5}$ & $2.44 \cdot 10^{-6}$ \\
0.5 & 0.1 & 0.1 & 2 & & $3.15 \cdot 10^{-5}$ \\
0.5 & 0.1 & 0.1 & 3 & & $1.24 \cdot 10^{-5}$ \\
\hline
\end{tabular}

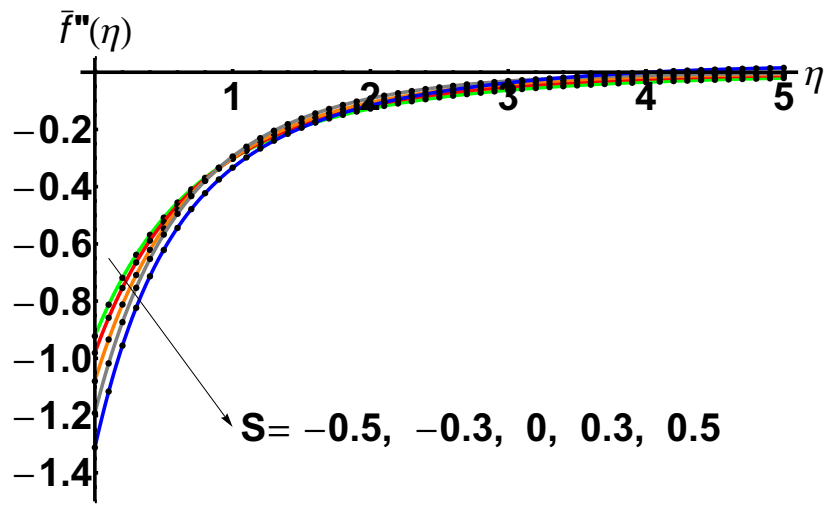

Figure 4: Variation of the shear stress $\bar{f}^{\prime \prime}(\eta)$ with increasing of the suction/blowing parameter $S$ for $\lambda=0.1$ :

— OHAM solution ; $\bullet \bullet \bullet \bullet \bullet \cdot$ numerical solution

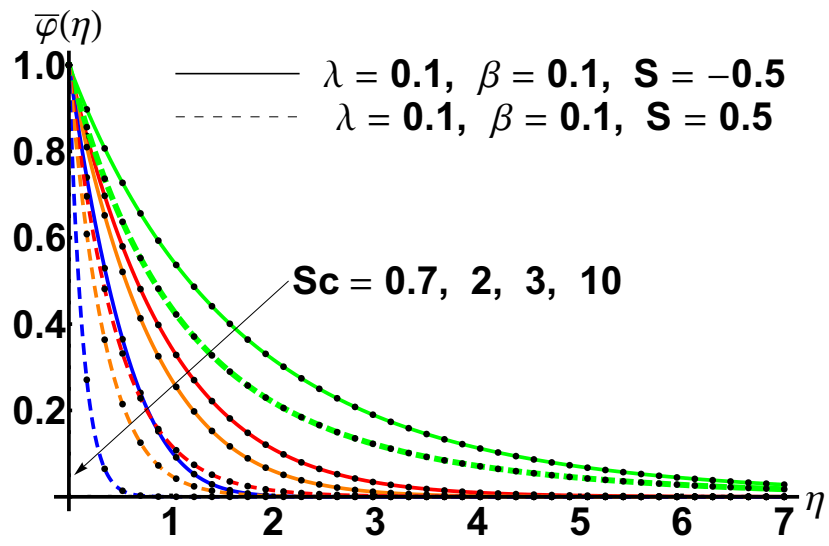

Figure 5: Variation of the concentration $\bar{\varphi}(\eta)$ with increasing of the Schmidt number $S c$ for $\lambda=0.1, \beta=0.1$ and different values of the suction/blowing parameter $S: \bullet \cdots \cdots \bullet$ numerical solution ; $\mathrm{OHAM}$ solution (with lines and dashing lines respectively)

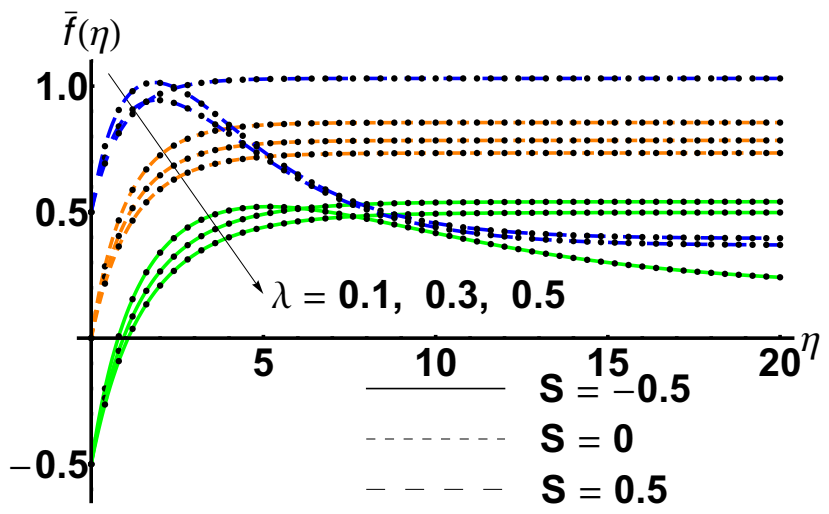

Figure 6: Variation of the stream function $\bar{f}(\eta)$ with increasing of the slip parameter $\lambda$ for different values of the suction/blowing parameter $S: \bullet \cdots \cdots \cdots$ numerical solution ; OHAM solution (with lines and dashing lines respectively)

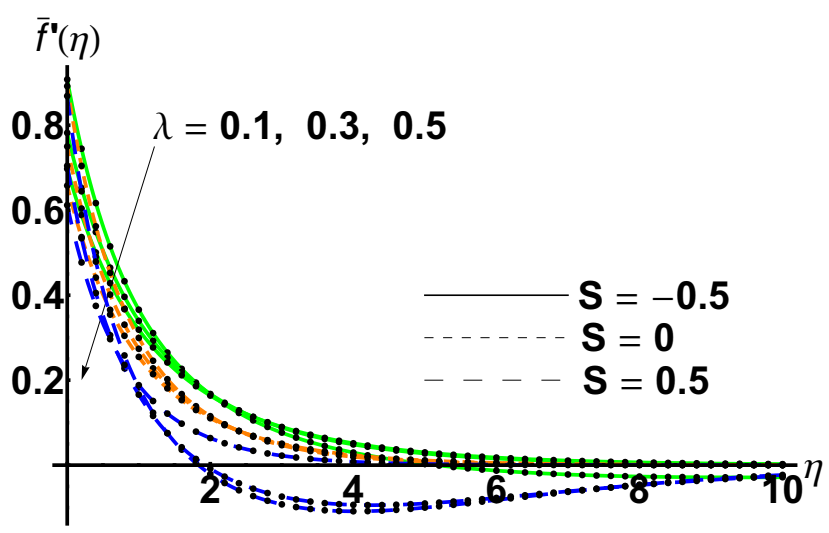

Figure 7: Variation of the horizontal velocity $\bar{f}^{\prime}(\eta)$ with increasing of the slip parameter $\lambda$ for different values of the suction/blowing parameter $S: \bullet \cdots \cdots \cdots$ numerical solution ; OHAM solution (with lines and dashing lines respectively)

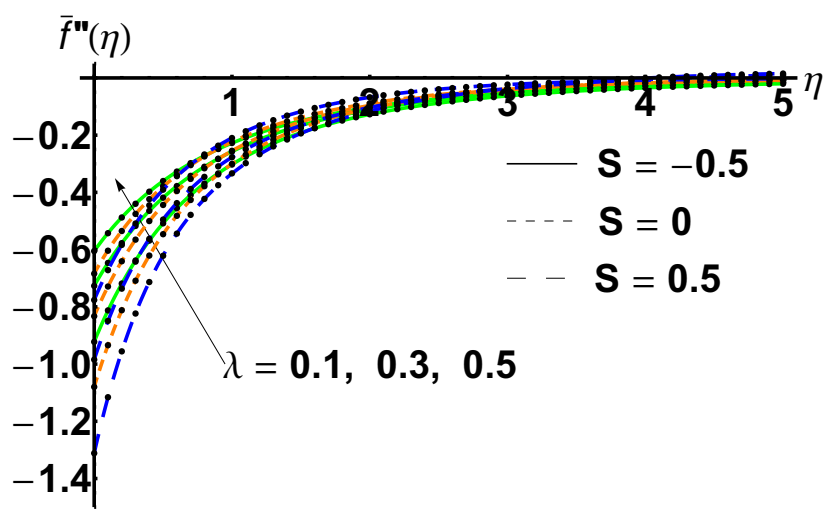

Figure 8: Variation of the shear stress $\bar{f}^{\prime \prime}(\eta)$ with increasing of the slip parameter $\lambda$ for different values of the suction/blowing parameter $S: \bullet \bullet \bullet \bullet \bullet \bullet$ numerical solution ; OHAM solution (with lines and dashing lines respectively) 


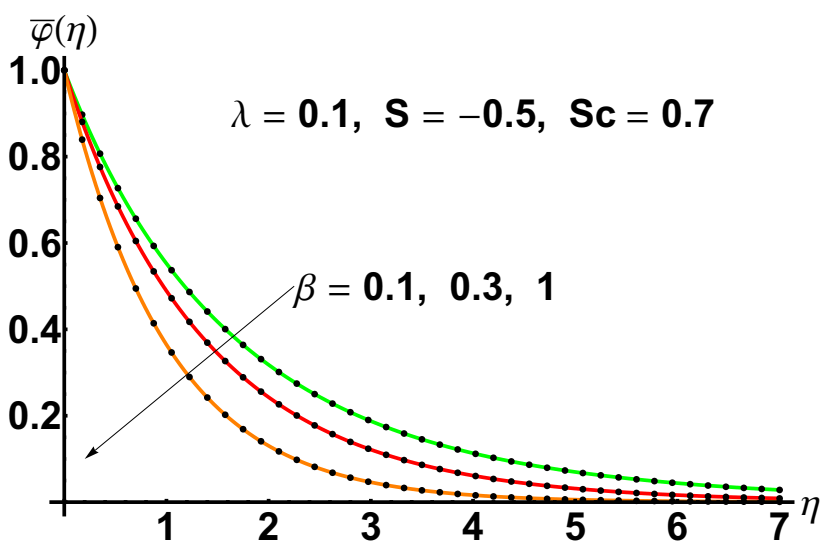

Figure 9: Variation of the concentration $\bar{\varphi}(\eta)$ given by Eqs. (53), (54) and (55) with increasing of the reaction rate parameter $\beta$ for $S=-0.5, \lambda=0.1$ and $S c=0.7:-$ OHAM solution $; \bullet \bullet \bullet \bullet \bullet \bullet \bullet$ numerical solution

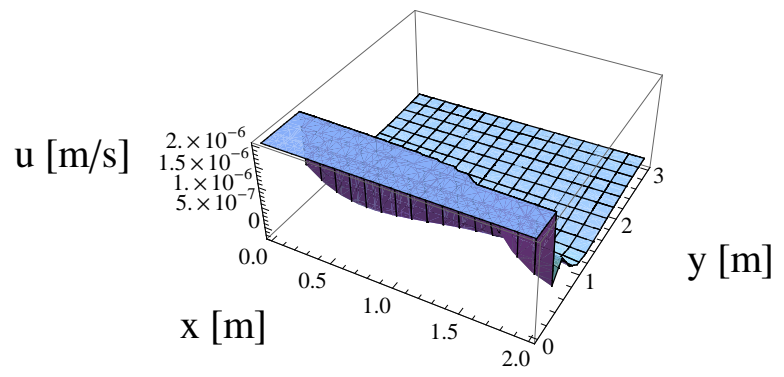

Figure 10: Variation of the velocity component $u$ from Eq. (4) for $\lambda=0.1, S=-0.3$, for hydraulic oil at a temperature of $40^{\circ} \mathrm{C}$.

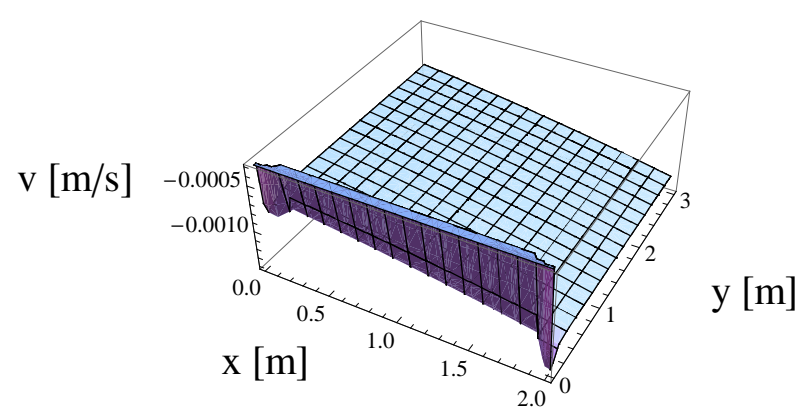

Figure 11: Variation of the velocity component $v$ from Eq. (4) for $\lambda=0.1, S=-0.3$, for hydraulic oil at a temperature of $40^{\circ} \mathrm{C}$.

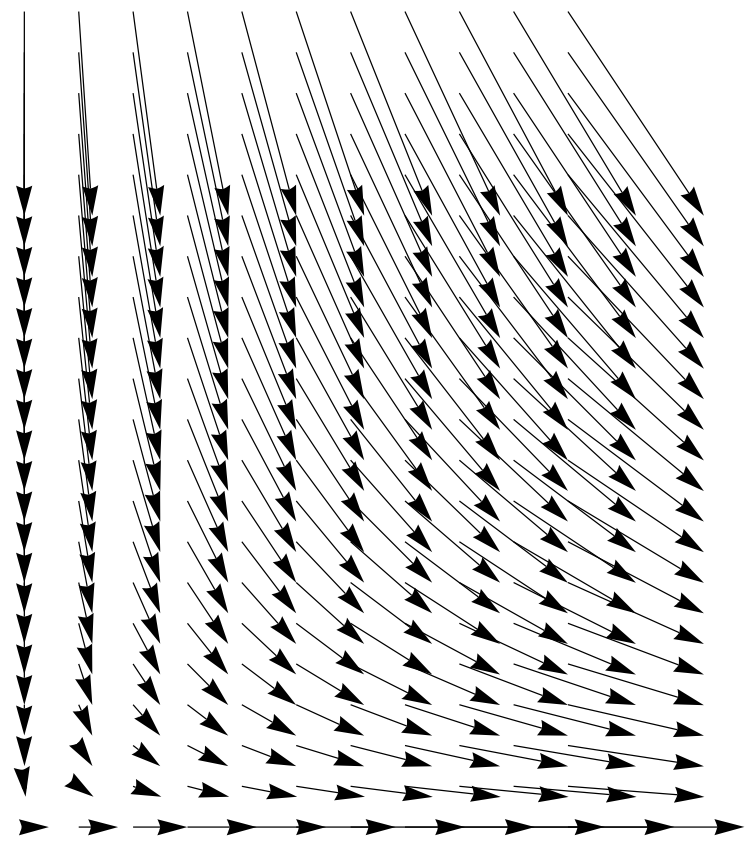

Figure 12: The vector field $(u, v)$ from Eq. (4) for $\lambda=0.1, S=-0.3$, for hydraulic oil at a temperature of $40^{\circ} \mathrm{C}$.

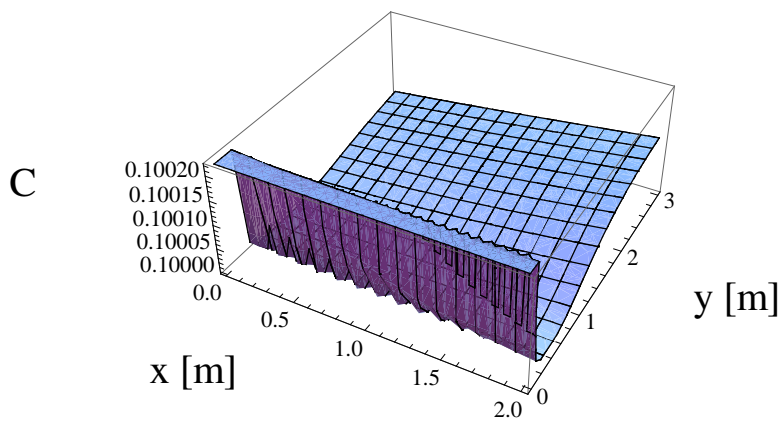

Figure 13: The concentration $C$ from Eq. (4) for $\lambda=0.1, S=-0.3$, $S c=2, \beta=0.1$ for hydraulic oil at a temperature of $40^{\circ} \mathrm{C}$. 